\title{
Pressure image assimilation for atmospheric motion estimation
}

\author{
By Thomas Corpetti ${ }^{1,5 \star}$, Patrick Héas ${ }^{2}$, Etienne Mémin ${ }^{1,3}$, Nicolas Papadakis ${ }^{4}$, \\ ${ }^{1}$ IRISA/INRIA - Campus de Beaulieu - 35042 Rennes cedex - France \\ ${ }^{2}$ CEMAGREF - Avenue de Cucillé- 35044 Rennes cedex - France \\ ${ }^{3}$ Fac. de Ing. de la Univ. Buenos-Aires, Av. Paseo Colón 850, C1063ACV Buenos Aires - Argentina \\ ${ }^{4} U P F$, Barcelona Media, Carrer Ocata 1, 08003 Barcelona - Spain \\ ${ }^{5}$ CNRS / COSTEL UMR 6554, Place du Recteur Henri Le Moal, 35043 Rennes Cedex - France
}

September 1, 2008

\begin{abstract}
The complexity of the laws of dynamics governing 3D atmospheric flows associated with incomplete and noisy observations make the recovery of atmospheric dynamics from satellite image sequences very difficult. In this paper, we address the challenging problem of estimating physical sound and time-consistent horizontal motion fields at various atmospheric depths for a whole image sequence. Based on a vertical decomposition of the atmosphere, we propose a dynamically consistent atmospheric motion estimator relying on a multi-layer dynamic model. This estimator is based on a weak constraint variational data assimilation scheme and is applied on noisy and incomplete pressure difference observations derived from satellite images. The dynamic model is a simplified vorticity-divergence form of a multi-layer shallow-water model. Average horizontal motion fields are estimated for each layer. The performance of the proposed technique is assessed using synthetic examples and using real world meteorological satellite image sequences. In particular, it is shown that the estimator enables exploiting fine spatio-temporal image structures and succeeds in characterizing motion at small spatial scales.
\end{abstract}

\section{Introduction}

\subsection{Overview}

Geophysical motion characterization and image sequence analysis are crucial issues for numerous scientific domains involved in the study of climate change, weather forecasting and climate prediction or biosphere analysis. The use of surface station, balloon or in-flight aircraft measurements has improved the estimation of wind fields and has been a further step for an improved understanding of meteorological phenomena. However, the network's temporal and spatial resolutions may be insufficient for the analysis of mesoscale dynamics. Recently, in an effort to avoid these limitations, high-resolution satellites sensors have been designed to provide image sequences characterized by finer spatial and temporal resolutions. Nevertheless, the analysis of atmospheric motion from image sequences remains particularly challenging due to the complexity of atmospheric dynamics observed at such scales. Thus, advanced techniques are needed to exploit the latest generation of satellite images.

\footnotetext{
* Corresponding author.

e-mail: thomas.corpetti@irisa.fr
}

\subsection{Related works}

For image-based geophysical motion analysis standard techniques from computer vision, originally designed for bidimensional quasi-rigid motions with stable salient features, are not well adapted (Leese et al., 1971; Horn and Schunck, 1981). The design of techniques specific to fluid flows has been a step forward, towards the elaboration of reliable methods to extract characteristic features of flows (Zhou et al., 2000; Corpetti et al., 2002; 2006; Cuzol et al., 2007; Cuzol and Mémin, 2008; Yuan et al., 2007). However, for geophysical applications, existing fluid-dedicated methods are all limited to frame-to-frame estimation and do not rely on physical conservation laws.

Geophysical flows are quite well described by appropriate physical models. As a consequence, in such contexts, the inclusion of physical evolution laws should constitute a very powerful means for the motion analysis of satellite image data, when compared with standard variational or statistical generic image based motion estimation techniques.

Recently, a layered motion estimator based on the shallow-water mass conservation equation has been proposed in (Héas et al., 2007; Héas and Mémin, 2008). In these studies, time consistency is reinforced by the introduction of a frame-to-frame temporal regularization based on a simplified vorticity-divergence form of the shallow-water momentum conservation equations. This dynamic model provides an additional constraint guiding the estimation process from 
the results obtained using the previous frames. Nevertheless, it is important to outline that this constraint is not imposed globally on the sequence of observations but only on a pair of images.

Variational data assimilation (Le Dimet and Talagrand, 1986; Talagrand and Courtier, 1987), derived from optimal control theory (Lions, 1971), offers a global optimal formulation allowing the combination of physical models and different kinds of observations. Since its introduction, the variational assimilation technique, commonly known as 4D-Var, has been widely used for several atmospheric applications (Bennet, 1992; Talagrand, 1997) and especially for numerical weather forecasting or climate numerical modeling (Bennet et al., 1996; Courtier et al., 1994; Courtier and Talagrand, 1990; Ghil and Malanotte-Rizzoli, 1991; Le Dimet and Blum, 2002; Rabier et al., 1998). Different variations of the original principles have been proposed over the last 15 years and this technique is routinely used in several operational meteorological centers. The exploitation of the spatial information of satellite data have been shown to be very promising for improving Numerical Weather Prediction (NWP - see for instance some applications concerning assimilation of topclouds in (Bayler et al., 2000; Benjamin et al., 2002; Kim and Benjamin, 2000) or radiances in (Andersson et al., 1994)).

Image observations possess very good spatial resolutions compared to spatial scales of standard data and are largely used in assimilation systems. However, only a few attempts have been made to date to consider their use for the direct estimation of dynamic parameters (like the wind fields) that are indirectly observed through the image sequences. Even if some assimilation procedures are able to capture a wind field (viewed principally as a control parameter of a dynamic system like in (Andersson et al., 1994)), none of them assimilate this quantity directly through an observation operator linking the motion to the image data. This issue is indeed particularly difficult due to the phenomenon responsible for the image formation and to the inherent projection on the image plane of the three-dimensional observed quantity. As a consequence, the operational estimation of wind fields is currently done using correlation techniques, such as the Atmospheric Motion Vectors (AMV) product supplied by Eumetsat (the European agency that supplies the METEOSAT satellite data). The height is then determined from the infrared temperature and converted to pressure using an ECMWF forecast profile (Menzel et al., 1983; Holmlund, 1998; Schmetz et al., 1993; 1995). Once estimated, these AMV can either be the input of data assimilation algorithms or be assimilated themselves. In this paper, we propose the suppression od the intermediary step of estimating AMV and to assimilate directly the wind field from the sequence of images. Indeed, as shown in a recent study on a turbulent 2D flow (Papadakis et al., 2007) the use of a direct image measurement operator clearly leads to a great improvement of the quality of the estimation compared to an assimilation process based on motion fields given by an external process (pseudo-observations of the Eulerian velocity fields).

Variational data assimilation techniques relying on image data or on features extracted from image data have recently been proposed. A technique for the assimilation of a low order dynamic system, obtain from a POD-Galerkin projection, has been proposed in (D'Adamo et al., 2007).
This technique makes use of noisy flow motion measurements estimated from a set of images of particles. In (Ruhnau and Schnörr, 2007), an optimal control strategy has also been proposed for the recovery of fluid motion. This approach based on a Stokes flow model nevertheless remains an estimation technique that works only on two consecutive frames and no dynamic coherency can be guaranteed along the image sequence. Taking a different approach, two variational assimilation strategies have been proposed in the computer vision community for the tracking of curves (Papadakis and Mémin, 2008) and the estimation of fluid motion fields (Papadakis et al., 2007). Both of these methods directly assimilate image data. They do not depend on pseudo-measurements obtained by external techniques but rather propose to directly include a differential observation operator borrowed to image features estimation techniques.

The technique proposed in this paper consists of an extension of these studies for atmospheric motion estimation from satellite image sequences.

\subsection{Contributions}

The method we propose differs significantly from previous stuidies on remote sensing motion analysis. In contrast to previous image-based techniques relying on two consecutive frames, the proposed approach estimates dynamically consistent motion fields through a whole image sequence.

The method exploits a simplified version of a divergence vorticity formulation of a shallow-water dynamic system. This inexact dynamics is complemented by the use of uncertainty terms. The assimilation is embedded into an incremental optimal control scheme and relies on sparse pressure data of atmospheric layers. The choice of this simplified dynamics is guided by the nature of the observations we have at our disposal. The efficiency of this process will be demonstrated using synthetic benchmarks and analyzed using real world satellite sequences.

This article is organized as follows. After a section describing the context of the study, the data and the main dynamic assumptions, the assimilation methodology is presented in section 3. Section 4 is devoted to the exploitation of this framework to the present problem. Experimental results, on synthetic and real examples, are provided in section 5. A summary, some perspectives as well as appendixes conclude this paper.

Before entering into the detailed description of the proposed technique, let us first situate more precisely the aims of our study, the assumptions inherited from these objectives and the nature of the available image data.

\section{Context, objectives and ensuing dynamic assumptions}

In this study we aim to recover as accurately as possible a dense description of horizontal wind fields in the 3D physical space. Nevertheless, as satellite images usually only provide sparse data of partially visible 3D cloud layers from which only inaccurate pressure vertical coordinates can be indirectly inferred, we will focus on the simpler task of estimating integrated horizontal wind fields for successive atmospheric layers. 


\subsection{Layers decomposition of the atmosphere}

The layering of atmospheric flow in the troposphere is valid as long as horizontal scales are much greater than the vertical scale height. Thus, for layers of about $1 \mathrm{~km}$ thick, this hypothesis is roughly valid for horizontal scales greater than or equal to $100 \mathrm{~km}$. It is thus impossible to truly characterize a layered atmosphere with a local analysis performed in the vicinity of a pixel representing a kilometer order scale. Nevertheless, one can still decompose the 3D space into elements of variable thickness, where only sufficiently thin regions of such elements may really correspond to common layers. An analysis based on such a decomposition has the principal advantage of operating at different atmospheric pressure ranges and avoids the mixing of heterogeneous observations.

The 3D space decomposition used here is one introduced in earlier articles (Héas et al., 2007; Héas and Mémin, 2008), where the $k$-th $(k \in[1, K])$ layer corresponds to the volume lying in between an upper surface $s^{k+1}$ and a lower surface $s^{k}$. These surfaces $s^{k+1}$ are defined by the height of the top of clouds belonging to the $k$-th layer. They are thus only defined in areas where clouds belonging to the $k$-th layer exist, and remain undefined elsewhere. Cloud classification maps, as illustrated in figure 1, determine the allocation of the top of clouds to the different layers. The EUMETSAT consortium routinely provides such classifications, which are based on thresholds of top of cloud pressure.

\subsection{Sparse pressure difference image observations}

Top of cloud pressure images of the order of one kilometer are also routinely provided by the EUMETSAT consortium $^{1}$. We denote by $C^{k}$ the class corresponding to the $k$-th layer. It should be noted that the top of cloud pressure image is composed of segments of top of cloud pressure functions $p\left(s^{k}\right)$ related to the different layers : $\left\{\bigcup_{k} p\left(x, y, s^{k+1} \mid(x, y) \in\right.\right.$ $\left.C^{k}\right\}$.

Thus, pressure images of top of clouds are used to constitute sparse pressure maps of the upper boundaries of the layers $p\left(s^{k+1}\right)$. As with satellite images the lower cloud boundaries are always occluded, we coarsely approximate the missing pressure observations $p\left(s^{k}\right)$ by an average pressure value $\bar{p}\left(s^{k}\right)$ observed on the top of the clouds of the layer underneath. Finally, for the layer $k \in[1, K]$, we define observations $h_{o b s}^{k}$ as pressure differences in hecto Pascal (hPa) units:

1 Top of cloud pressure images are derived from a radiative transfer model using ancillary data, namely temperature and humidity profiles obtained by analysis of short term forecasts. This model simulates the radiation by the top of an opaque cloud at different vertical levels, which might be observed by a satellite. The pressure level where the simulated radiation best fits with the observed radiation determines the pressure of the cloud top for the corresponding pixel (Lutz, 1999). Multi-channel techniques (using a thermal IR with a water vapor or $\mathrm{CO}_{2}$ absorption channels) enable the determination of the temperature of the top of semitransparent clouds (Menzel et al., 1983; Schmetz et al., 1993), and thus their equivalent pressure level (with the help of analyzed or forecast data) independently from foreground or background effects.

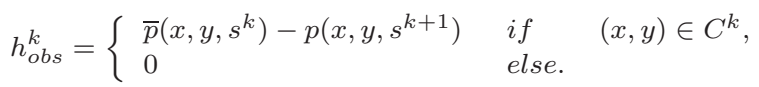

The resulting observations from a METEOSAT image (5-June-2004, 13h30 UTC -universal time, similar to GMT : Greenwich mean time- or 2004-06-05T13:30Z in ISO8601) are illustrated in figure 1 . The scattered nature of these maps (less than $15 \%$ is exploitable) is due to the sparsity of cloud coverage but is also enforced by the higher altitude clouds occultation of the lower layers. We should also note that the use of pressure images, besides being coherent with our layers definition, will enable the creation of a precise data model relating the observed pressure data to the unknown motion field. In contrast to traditional atmospheric wind field extraction techniques, we do not rely on the assumption that cloud luminance patterns are passive tracers of the flow (assumption which is known to be violated in some situations). This is a key advantage of the proposed approach.

\subsection{Ensuing dynamic assumptions}

In order to construct the best possible dynamic model describing the evolution of the pressure layers analyzed and in accordance with the objectives of this study (i.e. the recovering of the horizontal displacements of the set of layers), we rely on the shallow-water approximation to integrate vertically the horizontal primitive equations of atmospheric dynamics. Using the hypothesis of hydrostatic equilibrium, the atmospheric hydrostatic primitive equations read:

$$
\left\{\begin{aligned}
\frac{d u}{d t}-\frac{u v \tan \phi}{a}+\frac{u w}{a}+\frac{p_{x}}{\rho} & =+2 \Omega v \sin \phi-2 \Omega w \cos \phi+\mathcal{F}_{u} \\
\frac{d v}{d t}+\frac{u^{2} \tan \phi}{a}+\frac{v w}{a}+\frac{p_{y}}{\rho} & =-2 \Omega u \sin \phi+\mathcal{F}_{v} \\
\frac{d p}{d z}+\rho g & =0,
\end{aligned}\right.
$$

where $(u, v)$ represents the horizontal velocity field, $w$ its vertical component, $p$ the pressure $\left(p_{x}\right.$ and $p_{y}$ denoting the spatial derivatives w.r.t $x$ and $y),\left(\mathcal{F}_{u}, \mathcal{F}_{v}\right)$ the horizontal components of viscous forces at subgrid scales (Frisch, 1995), $\phi$ the latitude, $\rho$ the density, $\Omega$ and $a$ the Earth angular velocity and its radius. The continuity equation complements the system:

$$
\frac{1}{\rho} \frac{d \rho}{d t}+\nabla \cdot \mathbf{V}=0
$$

where $\mathbf{V}=(u, v, w)^{T}$ stands for the $3 \mathrm{D}$ velocity.

A vertical integration of the primitive equations is not straightforward since, in our case, we only rely on pressure image analysis and vertical density profiles remain unknown. We circumvent this difficulty by relying on a shallowwater approximation of these primitive equations (De SaintVenant, 1871).

The shallow-water approximation is based on two main assumptions 1) vertical motion is negligible with respect to horizontal motion and 2) incompressibility. For atmospheric dynamics, this is valid for the upper range of mesoscale analysis in a layered atmosphere. In the following section we derive a dynamic model using these two hypotheses.

2.3.1 Negligible influence of the vertical motion Considering horizontal scales of the order of $100 \mathrm{~km}$, combined with layer depths of the order of $1 \mathrm{~km}$, makes the shallowwater approximation relevant. Therefore, in order to obtain 
a valid dynamic model on a pixel grid, we filter the hydrostatic primitives equations with a Gaussian kernel function $K_{\delta_{x}}$ of standard deviation equal to $\delta_{x}=100 \delta_{p}^{-1}$, where $\delta_{p}$ denotes the image pixel resolution in kilometers ${ }^{2}$. Following the scale analysis presented in appendix A, where the Rossby number is around 0.5, Coriolis terms have to be included in the momentum components of the filtered hydrostatic primitive equations adapted to mesoscale dynamics. These latter equations are written:

$$
\begin{cases}\frac{d \tilde{u}}{d t}+\frac{\tilde{p}_{x}}{\rho}-\tilde{v} f^{\phi} & =\nu_{\mathcal{T}} \Delta \tilde{u} \\ \frac{d \tilde{v}}{d t}+\frac{p_{y}}{\rho}+\tilde{u} f^{\phi} & =\nu_{\mathcal{T}} \Delta \tilde{v},\end{cases}
$$

where we have introduced the filtered pressure $\tilde{p}=K_{\delta_{x}} * p$, the filtered horizontal wind $\tilde{\mathbf{v}}=(\tilde{u}, \tilde{v})^{T}=K_{\delta_{x}} *(u, v)^{T}$ and where $\rho_{0}, f^{\phi}$ and $\nu_{\mathcal{T}}$ denote the local mean density, the Coriolis factor depending on latitude $\phi$ and the turbulent viscosity produced at sub-grid scales. The induced turbulent dissipation can be approached by sub-grid models proposed in Large Eddy Simulation literature (Meneveau and Katz, 2000). The simplest one is the well known Smagorinsky subgrid model which is in agreement with Kolmogorov "K41" theory (Smagorinsky, 1963). For 2D flows, it results in an anisotropic diffusion with a turbulent viscosity coefficient equal to :

$$
\nu_{\mathcal{T}}=\left(C \delta_{x}\right)^{2} \sqrt{2\left(\tilde{u}_{x}^{2}+\tilde{v}_{y}^{2}+\left(\tilde{u}_{y}+\tilde{v}_{x}\right)^{2}\right)},
$$

where $C$ is the Smagorinsky coefficient (which is usually fixed to 0.17 ).

2.3.2 Incompressibility The shallow-water approximation also assumes incompressibility. Thus, we consider a constant density $\rho_{0}^{k}$ within any layer $k$ ( $k$ is the index related to the number of the layer). We should note that this incompressibility simplification which underlies the shallow-water modeling is reasonable in our case, while it may be erroneous for finer horizontal scales. Layer average densities $\rho_{0}^{k}$ can be related to the average pressures $\bar{p}^{k}$ by vertical integration of the equation of state for dry air $(p=\rho R T)$, combined with the hydrostatic relation $(d p=-g \rho d z)$, using the assumption of constant lapse rate $\left(T=T_{0}+\gamma z\right)$, where $g, R, T_{0}$ and $\gamma$ denote the standard physical constants. Indeed, as demonstrated in appendix $\mathrm{B}$, the mean density related to the $k$-th layer may be expressed as :

$$
\rho_{0}^{k}=\frac{p_{0}^{2}}{\left(\bar{p}^{k+1}-\bar{p}^{k}\right)\left(\frac{\gamma R}{g}+2\right) R T_{0}}\left[\left(\frac{p}{p_{0}}\right)^{\frac{\gamma R}{g}+2}\right]_{\bar{p}^{k}}^{\bar{p}^{k+1}} .
$$

This relation characterizes a barotropic atmosphere (Holton, 1994). Expanding the total derivatives in the isobaric coordinate system $(x, y, p)$ and using the fact that the flow is incompressible (zero local 3D divergence), equation (4) can be rewritten as:

$$
\left\{\begin{array}{lll}
\frac{\partial \tilde{u}}{\partial t}+\frac{\partial \tilde{u}^{2}}{\partial x}+\frac{\partial \tilde{u} \tilde{v}}{\partial y}+\frac{\partial \tilde{u} \tilde{w}}{\partial p}+\frac{\tilde{p}_{x}}{\rho_{0}}-\tilde{v} f^{\phi} & = & \nu_{\mathcal{T}} \Delta \tilde{u} \\
\frac{\partial \tilde{v}}{\partial t}+\frac{\partial \tilde{u} \tilde{v}}{\partial x}+\frac{\partial \tilde{v}^{2}}{\partial y}+\frac{\partial \tilde{v} \tilde{w}}{\partial p}+\frac{\tilde{p}_{y}}{\rho_{0}}+\tilde{u} f^{\phi} & = & \nu_{\mathcal{T}} \Delta \tilde{v}
\end{array}\right.
$$

2 The Gaussian filtering operation is achieved by a convolution product with a Gaussian kernel and acts as a low-pass frequency filter: frequencies higher than a given value (which depends on the standard deviation) are removed. Its effect on the HPE equations is to keep only the "low-pass" component, i.e. the large scale structures. where $\tilde{\omega}=\frac{d \tilde{p}}{d t}$ denotes the filtered vertical wind component in pressure coordinates. In addition, the expression of the continuity equation (3) in the isobaric coordinate system $(x, y, p)$ yields the filtered relation:

$$
\frac{\partial \tilde{u}}{\partial x}+\frac{\partial \tilde{v}}{\partial y}+\frac{\partial \tilde{w}}{\partial p}=0
$$

where $(\tilde{u}, \tilde{v}, \tilde{w})$ are the components of the filtered 3D velocity.

We now derive the integrated shallow-water model dedicated to atmospheric layers ${ }^{3}$. In order to perform the vertical integration of equation (3) and equation (7) in the pressure interval $\left[\tilde{p}\left(s^{k+1}\right), \tilde{p}\left(s^{k}\right)\right]$, we first fix the boundary conditions:

$$
\begin{cases}\frac{\partial \tilde{p}\left(s^{k}\right)}{\partial t}+\tilde{u}\left(s^{k}\right) \frac{\partial \tilde{p}\left(s^{k}\right)}{\partial x}+\tilde{v}\left(s^{k}\right) \frac{\partial \tilde{p}\left(s^{k}\right)}{\partial y} & =\tilde{\omega}\left(s^{k}\right), \\ \frac{\partial \tilde{p}\left(s^{k+1}\right)}{\partial t}+\tilde{u}\left(s^{k+1}\right) \frac{\partial \tilde{p}\left(s^{k+1}\right)}{\partial x}+\tilde{v}\left(s^{k+1}\right) \frac{\partial \tilde{p}\left(s^{k+1}\right)}{\partial y} & =\tilde{\omega}\left(s^{k+1}\right) .\end{cases}
$$

Such boundary conditions can be interpreted as the fact that boundary surfaces $\tilde{p}\left(s^{k}\right)$ and $\tilde{p}\left(s^{k+1}\right)$ are deformed by the vertical wind $\tilde{\omega}\left(s^{k}\right)$ and $\tilde{\omega}\left(s^{k+1}\right)$ in a barotropic atmosphere. To achieve such a vertical integration in the pressure interval $\left[\tilde{p}\left(s^{k+1}\right), \tilde{p}\left(s^{k}\right)\right]$ varying with spatial coordinates, we employ the Leibnitz formula with the previous boundary conditions. This formula, which is valid for any integrable and derivable function $f(x, p)$ and for any interval $[a(x), b(x)]$ with boundaries depending on $x$, reads:

$$
\begin{gathered}
\int_{a(x)}^{b(x)} \frac{\partial f(x, p)}{\partial x} d p= \\
\frac{\partial}{\partial x}\left(\int_{a(x)}^{b(x)} f(x, p) d p\right)-f(x, b(x)) \frac{\partial b(x)}{\partial x}+f(x, a(x)) \frac{\partial a(x)}{\partial x} .
\end{gathered}
$$

Relying on equations (9) and (10), the vertical integration of equation $(7)$ in the pressure interval $\left[\tilde{p}\left(s^{k+1}\right), \tilde{p}\left(s^{k}\right)\right]$ gives rise to a momentum conservation equation for the $k$-th atmospheric layer:

$$
\frac{\partial\left(\tilde{\mathbf{q}}^{k}\right)}{\partial t}+\operatorname{div}\left(\frac{1}{\tilde{h}^{k}} \tilde{\mathbf{q}}^{k} \otimes \tilde{\mathbf{q}}^{k}\right)+\frac{1}{2 \rho_{0}^{k}} \nabla_{x y}\left(\tilde{h}^{k}\right)^{2}+\left[\begin{array}{cc}
0 & -1 \\
1 & 0
\end{array}\right] f^{\phi} \tilde{\mathbf{q}}^{k}=\nu_{\mathcal{T}}^{k} \Delta\left(\tilde{\mathbf{q}}^{k}\right),
$$

with

$$
\begin{aligned}
\tilde{h}^{k} & =\tilde{p}\left(s^{k}\right)-\tilde{p}\left(s^{k+1}\right), \\
\tilde{\mathbf{v}}^{k} & =\left(\tilde{u}^{k}, \tilde{v}^{k}\right)=\frac{1}{\tilde{h}^{k}} \int_{\tilde{p}\left(s^{k+1}\right)}^{\tilde{p}\left(s^{k}\right)} \tilde{\mathbf{v}} d p, \\
\tilde{\mathbf{q}}^{k} & =\tilde{h}^{k} \tilde{\mathbf{v}}^{k},
\end{aligned}
$$$$
\operatorname{div}\left(\frac{1}{\tilde{h}^{k}} \tilde{\mathbf{q}}^{k} \otimes \tilde{\mathbf{q}}^{k}\right)=\left[\frac{\frac{\partial\left(\tilde{h}^{k}\left(\tilde{u}^{k}\right)^{2}\right)}{\partial x}+\frac{\partial\left(\tilde{h}^{k} \tilde{u}^{k} \tilde{v}^{k}\right)}{\partial y}}{\frac{\partial\left(\tilde{h}^{k} \tilde{u}^{k} \tilde{v}^{k}\right)}{\partial x}+\frac{\partial\left(\tilde{h}^{k}\left(\tilde{v}^{k}\right)^{2}\right)}{\partial y}}\right] .
$$

By vertical integration of the continuity equation (relation (8)) in the pressure interval $\left[\tilde{p}\left(s^{k+1}\right), \tilde{p}\left(s^{k}\right)\right]$, we supplement the momentum conservation law of equation (11) with the mass conservation law :

$$
\frac{\partial \tilde{h}^{k}}{\partial t}+\operatorname{div}\left(\tilde{\mathbf{q}}^{k}\right)=0
$$

3 Note that according to Fubini's theorem, if the motion fields are integrable then vertically averaged horizontally filtered fields are equivalent to horizontally filtered vertically averaged fields. 
and obtain the independent Saint-Venant equation system for any atmospheric layer $k \in[1, K]$ :

$$
\left\{\begin{array}{l}
\frac{\partial \tilde{h}^{k}}{\partial t}+\operatorname{div}\left(\tilde{\mathbf{q}}^{k}\right)=0, \\
\frac{\partial\left(\tilde{\mathbf{q}}^{k}\right)}{\partial t}+\operatorname{div}\left(\frac{1}{\tilde{h}^{k}} \tilde{\mathbf{q}}^{k} \otimes \tilde{\mathbf{q}}^{k}\right)+\frac{1}{2 \rho_{0}^{k}} \nabla_{x y}\left(\tilde{h}^{k}\right)^{2}+\left[\begin{array}{cc}
0 & -1 \\
1 & 0
\end{array}\right] f^{\phi} \tilde{\mathbf{q}}^{k} \\
=\nu_{\mathcal{T}}^{k} \Delta\left(\tilde{\mathbf{q}}^{k}\right) .
\end{array}\right.
$$

Note that as we are using the isobaric coordinate system, partial derivatives with respect to $x, y$ and $t$ are defined at constant pressure $p$. However, according to section 2.2, pressure difference observations $\tilde{h}_{o b s}^{k}$ correspond to rough constant pressure intervals. Therefore, such data fit the shallowwater model of equation (17) defined in the isobaric coordinate system. This model will be the basis of our assimilation process. Before describing the developed approach, the next section presents the data assimilation principle.

\section{Data assimilation}

In this section we present the main principles of variational data assimilation for an imperfect dynamic model. We refer the reader to (Bennet, 1992; Le Dimet and Talagrand, 1986; Lions, 1971; Talagrand and Courtier, 1987; Vidard et al., 2000) for complete methodological aspects of data assimilation and applications concerning geophysical flows.

Our problem consists of recovering, from an initial condition, a system's state $X$ partially observed and driven by approximately known dynamics. This can be formalized as finding $X(\mathbf{s}, t)$, for any location $\mathbf{s}$ at time $t \in\left[t_{0}, t_{f}\right]$, that satisfies the system:

$$
\begin{array}{r}
\frac{\partial X}{\partial t}(\mathbf{s}, t)+\mathbb{M}(X(\mathbf{s}, t))=\nu_{m}(\mathbf{s}), \\
X\left(\mathbf{s}, t_{0}\right)=X_{0}(\mathbf{s})+\nu_{n}(\mathbf{s}), \\
\mathcal{Y}(\mathbf{s}, t)=\mathbb{H}(X(\mathbf{s}, t))+\nu_{o}(\mathbf{s}, t),
\end{array}
$$

where $\mathbb{M}$ is the non-linear operator relative to the dynamics, $X_{0}$ is the initial vector at time $t_{0}$ and $\left(\nu_{n}, \nu_{m}\right)$ are (unknown) additive control variables relative to noise on the dynamics and the initial condition respectively. In addition, noisy measurements $\mathcal{Y}$ of the unknown state are available through the non-linear operator $\mathbb{H}$ up to $\nu_{o}$. To estimate the system's state, a common methodology is the minimization of the cost function $\mathcal{J}$ :

$$
\begin{aligned}
\mathcal{J}(X)= & \frac{1}{2} \int_{t_{0}}^{t_{f}}\left\|\mathcal{Y}-\mathbb{H}\left(X\left(\nu_{m}, \nu_{n}\right)\right)\right\|_{R^{-1}}^{2} d t \\
& +\frac{1}{2}\left\|X\left(\mathbf{s}, t_{0}\right)-X_{0}(\mathbf{s})\right\|_{B^{-1}}^{2} \\
& +\frac{1}{2} \int_{t_{0}}^{t_{f}}\left\|\frac{\partial X}{\partial t}(\mathbf{s}, t)+\mathbb{M}(X(\mathbf{s}, t))\right\|_{Q^{-1}}^{2} d t
\end{aligned}
$$

where we have introduced the information matrices $R, B, Q$ relative to the covariance of the errors $\left(\nu_{m}, \nu_{n}, \nu_{o}\right)$. The Mahalanobis distance that has been used reads $\|X\|_{A^{-1}}=$ $X^{T} A^{-1} X$. The evaluation of $X$ can be done by canceling the gradient $\delta \mathcal{J}_{X}(\theta)=\lim _{\beta \rightarrow 0} \frac{J(X+\beta \theta)-J(X)}{\beta}$ of (21). Unfortunately, the estimation of such gradient is in practice unfeasible for a large system's state since it would be necessary to compute perturbations along all the components of $X$. One way to cope with this difficulty is to write an adjoint formulation of the problem. To that end, the adjoint variables $\boldsymbol{\lambda}$ that express the errors of the dynamic model are introduced as:

$$
\boldsymbol{\lambda}=Q^{-1}\left(\frac{\partial X}{\partial t}+\mathbb{M}(X+\beta \theta)\right)
$$

Denoting

- $\left(\frac{\partial \mathbb{M}}{\partial X}\right)$ and $\left(\frac{\partial \mathbb{H}}{\partial X}\right)$ the linear tangent operators of $\mathbb{M}$ and $\mathbb{H}$ respectively ${ }^{4}$,

- $\left(\partial_{X} \mathbb{M}\right)^{*}$ and $\left(\partial_{X} \mathbb{H}\right)^{*}$ their adjoint operators ${ }^{5}$,

it can be shown that canceling the gradient $\delta \mathcal{J}_{X}(\theta)$ w.r.t the adjoint variables $\lambda$ leads to a retrograde integration of an adjoint evolution model that takes into account the observations. Once the adjoint variables $\boldsymbol{\lambda}$ are estimated, one can recover the system state $X$ using relation (22). Finally, recovering $X$ leads to the following incremental algorithm (Bennett and Thorburn, 1992):

(i) Starting from $\tilde{X}\left(\mathbf{s}, t_{0}\right)=X_{0}(\mathbf{s})$, perform a forward integration: $\frac{\partial \tilde{X}}{\partial t}+\mathbb{M}(\tilde{X})=0$

(ii) $\tilde{X}(\mathbf{s}, t)$ being available, compute the adjoint variables $\boldsymbol{\lambda}(\mathbf{s}, t)$ with the backward equation:

$$
\begin{aligned}
& \boldsymbol{\lambda}\left(t_{f}\right)=0 ; \\
& -\frac{\partial \boldsymbol{\lambda}}{\partial t}(t)+\left(\partial_{X} \mathbb{M}\right)^{*} \boldsymbol{\lambda}(t)=\left(\partial_{X} \mathbb{H}\right)^{*} R^{-1}(\mathcal{Y}-\mathbb{H}(\tilde{X}))(t)
\end{aligned}
$$

(iii) Update the initial condition : $d X\left(t_{0}\right)=B \boldsymbol{\lambda}\left(t_{0}\right)$;

(iv) $\boldsymbol{\lambda}$ being available, compute the state space $d X(t)$ from $d X\left(t_{0}\right)$ with the forward integration

$$
\frac{\partial d X}{\partial t}(t)+\left(\frac{\partial \mathbb{M}}{\partial \tilde{X}}\right) d X(t)=Q \boldsymbol{\lambda}(t)
$$

(v) Update : $\tilde{X}=\tilde{X}+d X$

(vi) Loop to step (ii) until convergence

Intuitively, the adjoint variables $\boldsymbol{\lambda}$ contain information about the discrepancy between the observations and the dynamic model. They are computed from a current solution $\tilde{X}$ with the backward integration (23) that encompasses both the observations and the dynamic operators. This deviation indicator between the observations and the model is then used to refine the initial condition (step (iii)) and to recover the system state through an imperfect dynamic model where errors are $Q \boldsymbol{\lambda}$ (step (iv)). It should be noted that if the dynamic is perfect, the associated error covariance $Q$ is zero and the algorithm only refines the initial condition. However from an image analysis point of view, a perfect modeling is difficult to obtain since the different models on which one can rely are usually inaccurate due, for instance, to 3D$2 \mathrm{D}$ projections, varying lighting conditions, completely unknown boundary conditions at the image boarders, etc.

\section{Data assimilation for multi-layer shallow-water model}

In this section, we propose to develop an image assimilation technique based on (17) to estimate the mesoscale flows.

\footnotetext{
4 The linear tangent of an operator $\mathbb{A}$ is the directional derivative of the operator (the Gâteaux derivative): $\lim _{\beta \rightarrow 0} \frac{\mathbb{A}(X+\beta \theta)-\mathbb{A}(X)}{\beta}$

5 The adjoint $\mathbb{A}^{*}$ of a linear operator $\mathbb{A}$ on a space $\mathcal{D}$ is such as $\forall x_{1}, x_{2} \in \mathcal{D},<\mathbb{A} x_{1}, x_{2}>=<x_{1}, \mathbb{A}^{*} x_{2}>$
} 
To that end, we further discuss in section 4.1 the shallowwater model and its limitations based on the available observations. The sections $4.2,4.3,4.4$ and 4.5 are respectively devoted to the detailed description of the system state, the selected dynamic model, the observation system and the definition of the different error covariance matrices.

\subsection{Dynamic Model}

4.1.1 Limitation of Saint Venant's shallow-water model The dynamic model of equation (17) describes accurately the mesoscale atmospheric flows. Therefore, one can immediately define an assimilation scheme relying on the system variables:

$$
X=\left[\begin{array}{c}
\tilde{h}^{k} \\
\tilde{\mathbf{q}}^{k}
\end{array}\right]
$$

where the perfect dynamic model is given by relation (17). Remembering that images of pressure differences, $h^{k}$, are observable, it is possible to define the measurement system $\mathcal{Y}=\mathbb{H}(X)$ as:

$$
\begin{cases}\mathcal{Y} & =\tilde{h}_{o b s}^{k}=K_{\delta_{x}} * h_{o b s}^{k} \\ \mathbb{H} & =\left[I_{d}, 0\right] .\end{cases}
$$

This system has the advantage of assimilating both the sparse pressure differences $\tilde{h}_{o b s}^{k}$ and the associated motion field $\tilde{\mathbf{q}}^{k}$ without observing this latter quantity. However, it is important to note that the proposed shallow-water system describes the dynamics of physical quantities under a layering assumption. In practice, our data come from the Meteosat Second Generation sensor where a pixel corresponds to a square of $3000 \times 3000 \mathrm{~m}^{2}$. Even considering a spatial convolution by a Gaussian kernel $K_{\delta_{x}}$, a scale analysis reported in appendix $\mathrm{C}$ shows that the term $\frac{1}{2 \rho_{0}^{k}} \nabla_{x y}\left(\tilde{h}^{k}\right)^{2}$ in (17) locally dominates. This is all the more true in areas where the layering assumption breaks down at the considered observation scale. In particular, this term dominates on the borders of segments in sparse pressure images, where a pixel may contain heterogeneous contributions from different pressure levels. This perfect model is hence too restrictive and is likely to generate some numerical instabilities. We consequently prefer to develop, from this shallow-water model (17), an imperfect dynamic system that describes the evolution of the flow while authorizing the layering assumption to be violated at some locations. This is the scope of the next section.

\subsubsection{Divergence-vorticity shallow-water dynamic model} In order to overcome the limitations mentioned above, we describe the evolution of the filtered state variables from a simplified version of the previously introduced shallowwater equations and perform assimilation using an imperfect modeling scheme. To that end, we assume filtered horizontal motion components to be homogeneous within the layer. In other words, we neglect their vertical derivatives and consider that filtered horizontal winds $\tilde{\mathbf{v}}^{k}$, which have been vertically averaged, are equal to filtered horizontal winds on layer upper surfaces $\mathbf{s}^{k+1}$. Using such an assumption yields :

$$
\tilde{\mathbf{v}}_{t}^{k}+\boldsymbol{\nabla}\left(\tilde{\mathbf{v}}^{k}\right) \tilde{\mathbf{v}}^{k}-\rho_{0}^{-1} \boldsymbol{\nabla} \tilde{p}\left(s^{k+1}\right)+\left[\begin{array}{cc}
0 & -1 \\
1 & 0
\end{array}\right] f^{\phi} \tilde{\mathbf{v}}^{k}=\nu_{\mathcal{T}} \boldsymbol{\Delta}\left(\tilde{\mathbf{v}}^{\mathbf{k}}\right),
$$

with the notations $\boldsymbol{\nabla}\left(\tilde{\mathbf{v}}^{\mathbf{k}}\right)=\left(\boldsymbol{\nabla} \tilde{u}^{k}, \boldsymbol{\nabla} \tilde{v}^{k}\right)^{\top}$ and $\boldsymbol{\Delta}\left(\tilde{\mathbf{v}}^{\mathbf{k}}\right)=$ $\left(\Delta \tilde{u}^{k}, \Delta \tilde{v}^{k}\right)^{\top}$. Let us denote the vorticity by $\tilde{\zeta}^{k}=\operatorname{curl}\left(\tilde{\mathbf{v}}^{k}\right)$ and the divergence by $\tilde{D}^{k}=\operatorname{div}\left(\tilde{\mathbf{v}}^{k}\right)$. The previous system may be expressed in its vorticity-divergence form:

$\left\{\begin{array}{l}\tilde{\zeta}_{t}^{k}+\tilde{\mathbf{v}}^{k} \cdot \nabla \tilde{\zeta}^{k}+\left(\tilde{\zeta}^{k}+f^{\phi}\right) \tilde{D}^{k}=\nu_{\mathcal{T}} \Delta\left(\tilde{\zeta}^{k}\right), \\ \tilde{D}_{t}^{k}+\tilde{\mathbf{v}}^{k} \cdot \nabla \tilde{D}^{k}+\left(\tilde{D}^{k}\right)^{2}-2|J|-\rho_{0}^{-1} \Delta \tilde{p}\left(s^{k+1}\right)+f^{\phi} \tilde{\zeta}^{k}=\nu_{\mathcal{T}} \Delta\left(\tilde{D}^{k}\right),\end{array}\right.$

where $|J|$ is the determinant of the Jacobian matrix of variables $\left(\tilde{u}^{k}, \tilde{v}^{k}\right)$. For vorticity based large eddy simulation formulations, we may rely on enstrophy-based sub-grid models (Mansour et al., 1978), instead of using the Smagorinsky model (equation (5)). This sub-grid dissipation model is based on Taylor's vorticity transfer and dissipation by small scales theory (Taylor, 1932) and reads:

$$
\nu_{\mathcal{T}}=\left(C \delta_{x}\right)^{2}\left|\tilde{\zeta}^{k}\right|
$$

In the momentum conservation formulations of equations (27) and (28), dynamic models predict the evolution of velocity components $\left(\tilde{u}^{k}, \tilde{v}^{k}\right)$ and of divergence and vorticity $\left(\tilde{\zeta}^{k}, \tilde{D}^{k}\right)$. In both models, one of the major difficulties is created by the dependence of the pressure variable $\tilde{p}\left(s^{k+1}\right)$ which is an unknown variable of the $k$-th layer state. Adding pressure as a new state variable driven by the mass conservation law (16) constitutes a solution for achieving the model integrations. However, in section 4.4, we will need this mass conservation law to define a proper observation operator. Thus, we search instead to derive a dynamic model which is independent of the pressure unknown. In contrast to the classical formulation, the vorticity-divergence equations have the advantage of providing such a model for the vorticity evolution. As regards the divergence, since at large scales, it can be considered weak almost everywhere, we will rely on an approximate evolution law. We assume here that the divergence is advected by the flow and a noise variable that encodes the uncertainty on the model. More precisely we will assume that the divergence map is a function of a stochastic process representing a particle position and is driven by the following stochastic differential equation:

$$
d \mathbf{x}(t)=\tilde{\mathbf{v}}^{k}(\mathbf{x}(t)) d t+\sqrt{2 \nu_{T}} d B(t) .
$$

This equation states that the particle position is known only up to an uncertainty that grows linearly with time. Here $B_{t}$ denotes a standard Brownian motion of $\mathrm{R}^{2}$. The process $\mathbf{x}_{t}$ starts at point, $\boldsymbol{x}_{o}$. It can be shown through the Ito formula and Kolmogorov's forward equation, that the expectation at time $t$ of such a function, $\xi(t, \mathbf{x})=\mathbb{E}\left[\operatorname{div} \tilde{\mathbf{v}}^{k}(\mathbf{x}(t))\right]$ follows an advection diffusion equation (Oksendal, 1998):

$$
\begin{aligned}
& \xi_{t}+\tilde{\mathbf{v}}^{k} \cdot \boldsymbol{\nabla} \xi+\xi \operatorname{div} \tilde{\mathbf{v}}^{k}-\nu_{T} \Delta \xi=0, \\
& \xi\left(0, \boldsymbol{x}_{0}\right)=\operatorname{div} \tilde{\mathbf{v}}^{k}\left(\boldsymbol{x}_{0}\right) .
\end{aligned}
$$

Assuming that the divergence of the flow is given by its expectation $\left(\tilde{D}^{k} \approx \xi\right)$ allows us to write the simplified filtered vorticity-divergence model for the layer $k$ as:

$\left\{\tilde{\zeta}_{t}^{k}+\tilde{\mathbf{v}}^{k} \cdot \nabla \tilde{\zeta}^{k}+\left(\tilde{\zeta}^{k}+f^{\phi}\right) \tilde{D}^{k}=\nu_{\mathcal{T}} \Delta \tilde{\zeta}^{k}\right.$, $\left\{\tilde{D}_{t}^{k}+\tilde{\mathbf{v}}^{k} \cdot \nabla \tilde{D}^{k}+\left(\tilde{D}^{k}\right)^{2}=\nu_{\mathcal{T}} \Delta \tilde{D}^{k}\right.$.

In this model we assume that the divergence of the flow is weak and is similar to the divergence expectation. The divergence equation no longer describes the evolution of the 
flow divergence but the evolution of its expectation. The expectation of the divergence value is advected by the flow and dissipates due to a subgrid isotropic uncertainty. This hypothesis is quite natural in large scale modeling.

\subsection{State variables}

We choose to represent the system state $X$ (i.e. the velocity field) through the curl and divergence components:

$$
X=\left[\begin{array}{c}
\tilde{\zeta}^{k} \\
\tilde{D}^{k}
\end{array}\right]
$$

as their evolution can be described by equation (32) and since they completely determine the underlying $2 \mathrm{D}$ velocity up to a harmonic transportation component. Denoting the orthogonal gradient by $\nabla^{\perp}=(-\partial / \partial y, \partial / \partial x)^{\top}$ and the 2D Green kernel $G$ associated with the Laplacian operator $\left(G(\cdot)=\frac{1}{2 \pi} \log (\cdot)\right)$, the Helmholtz decomposition of the field can be expressed as :

$$
\tilde{\mathbf{v}}^{k}=\underbrace{\boldsymbol{\nabla}^{\perp}\left(G * \tilde{\zeta}^{k}\right)}_{\tilde{\mathbf{v}}_{\text {sol }}^{k}}+\underbrace{\boldsymbol{\nabla}\left(G * \tilde{D}^{k}\right)}_{\tilde{\mathbf{v}}_{i r r}^{k}}+\tilde{\mathbf{v}}_{h a r}^{k}
$$

where $\tilde{\mathbf{v}}_{h a r}^{k}$ is a harmonic transportation part $\left(\operatorname{div} \tilde{\mathbf{v}}_{h a r}^{k}=\right.$ $\operatorname{curl} \tilde{\mathbf{v}}_{\text {har }}^{k}=0$ ). In our applications, we will assume that this component of the velocity is stationary within the temporal interval of the analyzed image sequence. This component may be recovered by subtracting its solenoidal and irrotationnal parts from the initial field $\tilde{\mathbf{v}}_{0}^{k}$. As a consequence, the field can be represented by its div-curl components as :

$$
\tilde{\mathbf{v}}^{k}=\boldsymbol{\nabla}^{\perp}\left(G * \tilde{\zeta}^{k}\right)+\boldsymbol{\nabla}\left(G * \tilde{D}^{k}\right)=\underbrace{\left[\boldsymbol{\nabla}^{\perp} G *, \boldsymbol{\nabla} G *\right]}_{\mathbb{H}_{G}} \underbrace{\left[\begin{array}{c}
\tilde{\zeta}^{k} \\
\tilde{D}^{k}
\end{array}\right]}_{X},
$$

where the operator $\mathbb{H}_{G}$ can efficiently be computed in the Fourier domain.

\subsection{Dynamic operator and its adjoint}

The dynamic model required for the assimilation in (18) is defined by relation (32). This model is associated with an imperfect dynamic modeling where the model errors are here related to the Coriolis effect, to the pressure difference dissipation of the divergence component and to the sub-grid stress tensor error of the vorticity and divergence component.

The associated tangent linear system (equation (24)) around the current solution $X^{\star}=\left[\tilde{\zeta}^{\star}, \tilde{D}^{\star}\right]^{T}$ is:

$$
\begin{aligned}
\partial_{t}\left[\begin{array}{c}
\tilde{\zeta}^{k} \\
\tilde{D}^{k}
\end{array}\right]+\underbrace{\left[\begin{array}{rr}
\tilde{\mathbf{v}}^{\star} \cdot \boldsymbol{\nabla}+\tilde{D}^{\star}-\nu_{\mathcal{T}} \Delta & \begin{array}{c}
\tilde{\zeta}^{\star}+f^{\phi} \\
0
\end{array} \\
\tilde{\mathbf{v}}^{\star} \cdot \boldsymbol{\nabla}^{\star}+\tilde{D}^{\star}-\nu_{\mathcal{T}} \Delta
\end{array}\right]}_{\partial_{X \star \mathbb{M}}}\left[\begin{array}{c}
\tilde{\zeta}^{k} \\
\tilde{D}^{k}
\end{array}\right] \\
=Q^{k} \lambda,
\end{aligned}
$$

where $\nu_{\mathcal{T}}=\left(C \delta_{x}\right)^{2}\left|\tilde{\zeta}^{\star}\right|$. This equation includes an error covariance matrix $Q^{k}$ that will be further defined in section 4.5. At each time increment $d t$, the current velocity solution $\tilde{\mathbf{v}}^{\star}$ is updated according to the vorticity $\tilde{\zeta}^{k}$, the divergence
$\tilde{D}^{k}$ and the harmonic transportation $\tilde{\mathbf{v}}_{h a r}^{k}$ using the operator defined in equation (35). The adjoint variables $\left[\lambda_{\zeta}^{k}, \lambda_{D}^{k}\right]^{T}$ are obtained through the following adjoint system:

$$
\left\{\begin{array}{l}
\lambda_{\zeta}^{k}\left(t_{f}\right)=0, \\
\lambda_{D}^{k}\left(t_{f}\right)=0, \\
-\partial_{t}\left[\begin{array}{c}
\lambda_{\zeta}^{k} \\
\lambda_{D}^{k}
\end{array}\right]+\underbrace{\left[\begin{array}{cc}
-\tilde{\mathbf{v}}^{\star} \cdot \boldsymbol{\nabla}+\tilde{D}^{\star}-\nu_{\mathcal{T}} \Delta & \tilde{\zeta}^{\star}+f^{\phi} \\
0 & -\tilde{\mathbf{v}}^{\star} \cdot \boldsymbol{\nabla}^{\star}+\tilde{D}^{\star}-\nu_{\mathcal{T}} \Delta
\end{array}\right]}_{\left(\partial_{X} \star \mathbb{M}\right)^{*}}\left[\begin{array}{c}
\lambda_{\zeta}^{k} \\
\lambda_{D}^{k}
\end{array}\right] \\
=\partial_{X^{k}} \mathbb{H}^{*} R^{-1}(Y-\mathbb{H}(X)) .
\end{array}\right.
$$

We should note that the turbulent viscosity $\nu_{\mathcal{T}}$ and the velocity $\tilde{\mathbf{v}}^{\star}$ are computed during the direct integration and maintained in the backward integration. The discretization of the bi-dimensional advection system must be done carefully to prevent numerical explosions. In this application, it has been achieved using the non-oscillatory schemes proposed in (Xu and Shu, 2006). The temporal integration has been implemented through a third order Runge-Kutta scheme and the time step $d t$ is defined in order to respect the total variation diminishing property (Kurganov and Tadmor, 2000). Once the direct dynamic model has been discretized, the matrix $\left(\partial_{X} * \mathbb{M}\right)$ is available and the adjoint system involved in (37) corresponds to the transpose of this matrix. More details about the construction of adjoint models can be found in (Talagrand and Courtier, 1987).

The observation components $\mathcal{Y}=\mathbb{H}(X)$ and the error covariance matrices are defined in the next sections.

\subsection{Image observation operator}

4.4.1 Extracting motion fields from pressure images In order to define an observation operator that links the unknown motion field $\tilde{\mathbf{v}}^{k}$ to images of pressure differences $h_{o b s}^{k}(\mathbf{s}, t)$, we use the mass conservation law of equation (16):

$$
\frac{\partial h_{o b s}^{k}(\mathbf{s}, t)}{\partial t}+\nabla h_{o b s}^{k}(\mathbf{s}, t) \cdot \tilde{\mathbf{v}}^{k}(\mathbf{s}, t)+h_{o b s}^{k}(\mathbf{s}, t) \operatorname{div} \tilde{\mathbf{v}}^{k}(\mathbf{s}, t) \approx 0,
$$

However, this formulation alone cannot be used to estimate $\tilde{\mathbf{v}}^{k}$ as it provides only one equation for two unknowns at each spatio-temporal location $(\mathbf{s}, t)$. To remove such ambiguities, a common approach is to assume a spatial coherence of wind field estimates in a given neighborhood (similar to the well-known approach of (Lucas and Kanade, 1981) used in computer vision). In the present case, this assumption is coherent since unknowns are velocity vectors $\tilde{\mathbf{v}}^{k}(\mathbf{s}, t)$ that are spatially filtered within a neighborhood of size $\delta_{x}$. The measured motion field should thus obey the following constraint:

$$
K_{\delta_{x}} *\left(\frac{\partial h_{o b s}^{k}}{\partial t}+\nabla h_{o b s}^{k} \cdot \tilde{\mathbf{v}}+h_{o b s}^{k} \operatorname{div} \tilde{\mathbf{v}}^{k}\right) \approx 0,
$$

where $K_{\delta_{x}}$ is the Gaussian kernel of standard deviation $\delta_{x}$. We should note that in practice, to prevent the influence of areas without any data in the border of the regions of interest $C^{k}$, all the points without pressure information have been removed from the Gaussian smoothing. From the relation (39) and recalling that $\tilde{\mathbf{v}}^{k}=\mathbb{H}_{G} X$ (with 
$\left.X=\left[\tilde{\zeta}^{k}, \tilde{D}^{k}\right]^{T}\right)$, one can easily define our observation system $\mathcal{Y}=\mathbb{H}(X)$ as:

$$
\left\{\begin{aligned}
\mathcal{Y}= & K_{\delta_{x}} * \frac{\partial h_{o b s}^{k}(\mathbf{s}, t)}{\partial t} \\
\mathbb{H}(X)= & -\left(K_{\delta_{x}} * \nabla h_{o b s}^{k}\right)^{T} \mathbb{H}_{G}(X) \\
& -\left(K_{\delta_{x}} * h_{o b s}^{k}\right) \nabla^{T} \mathbb{H}_{G}(X)
\end{aligned}\right.
$$

The observation operator $\mathbb{H}$ is linear w.r.t the state variable $X=\left[\tilde{\zeta}^{k}, \tilde{D}^{k}\right]^{T}$ and is then identical to its linear tangent operator. Noting that the adjoint of $\mathbb{H}_{G}$ is $-\mathbb{H}_{G}$ (as shown in (Papadakis and Mémin, 2007)), the adjoint $\partial_{X \star \mathbb{H}^{*}}(Y)$ of $\mathbb{H}(X)$ therefore reads :

$$
\begin{aligned}
\partial_{X \star \mathbb{H}^{*}}(Y)= & \mathbb{H}_{G}\left(\left(K_{\delta_{x}} * \nabla h_{o b s}^{k}\right) Y\right) \\
& -\mathbb{H}_{G}\left(\boldsymbol{\nabla}\left(\left(K_{\delta_{x}} * h_{o b s}^{k}\right) Y\right)\right)
\end{aligned}
$$

4.4.2 Observation retrieval and representativeness Most of the motion estimation techniques, which aim at extracting wind fields from satellite images (like AMV -Atmospheric Motion Vectors- from Eumetsat), are based on cloud luminance pattern matching over consecutive frames. Such techniques assume that clouds behave like tracers of the underlying motion field and that the cloud brightness function remains constant over time. It is unfortunately well known that these hypotheses are not always valid for satellite images (Corpetti et al., 2002; Schmetz et al., 1993). A worthwhile benefit of the present study is to dispense with these assumptions since the observed pressure differences are directly linked to the local Eulerian wind fields through a relation derived from the continuity equation.

However, these pressure difference maps have been obtained through a sequence of manipulations (cloud classification, conversion from pixel to temperature, from temperature to pressure) and simplifications (the pressure value of the unobserved "lower" level of the layer is arbitrarily assigned to a mean value). These manipulations are likely to generate some errors in the input data. These errors are quantifiable from Eumetsat products through 3 possible values of a quality parameter $\eta$ of the cloud height assignment. These values are set to $\eta(\mathbf{s})=0$ (no pressure in $\mathbf{s}$ ), $\eta(\mathbf{s})=1$ (pressure of good quality) or $\eta(\mathbf{s})=2$ (poor quality). This information will be taken into account for the definition of the error covariance matrices in the next section.

\subsection{Error covariance matrices and initialization issues}

4.5.1 Observations The inverse error covariance matrix related to the observations $\left(R^{k}\right)^{-1}$ is diagonal and defined using the mask of observation $C^{k}$ :

$$
\left(R^{k}\left(\mathbf{s}, \mathbf{s}^{\prime}\right)\right)^{-1}=\left\{\begin{array}{l}
\left(Z^{k}\right)^{-1}\left(P^{k}\right)^{-1} \text { if } \quad \mathbf{s}=\mathbf{s}^{\prime} \quad \text { and } \mathbf{s} \in C^{k} \\
0 \text { else. }
\end{array}\right.
$$

The inverse error covariance matrices $\left(Z^{k}\right)^{-1}$ and $\left(P^{k}\right)^{-1}$ are respectively related to a confidence measure related to the observation operator and on the pressure height assignment errors. We chose to define $\left(Z^{k}\right)^{-1}$ as

$$
\left(Z^{k}\left(\mathbf{s}, \mathbf{s}^{\prime}\right)\right)^{-1}=\alpha_{o b s}\left\{\begin{array}{l}
\exp \frac{-[\mathcal{Y}-\mathbb{H}(X)]^{2}}{\sigma^{2}} \text { if } \quad \mathbf{s}=\mathbf{s}^{\prime} \text { and } \mathbf{s} \in C^{k}, \\
0 \text { else. }
\end{array}\right.
$$

where $\sigma$ and $\alpha_{o b s}$ are parameters to be fixed. As shown in (Corpetti et al., 2008), this penalization amounts to considering a robust norm on the first term of the cost-function in (21). Such a robust function allows the discarding of points having large "residual" values of the observation error $[\mathcal{Y}-\mathbb{H}(X)]$ (called outliers in the Robust Statistics literature (Huber, 1981)). Compared to a quadratic cost function (i.e. $R=I_{d}$ ), the use of such robust semi-norms permits limiting the influence of those erroneous points. The robust norm, $\Phi(\cdot)$, is here expressed in an equivalent (with respect to its minimization) weighted quadratic expression in which the weight function involved, $z()=.\Phi^{\prime}(\sqrt{.})=\left(Z^{k}\right)^{-1}(x, y)$, is iteratively updated at every grid point. Different types of cost functions (convex or non convex) that obey this semiquadratic reformulation can be chosen under mere assumptions (mainly strict concavity of $\Phi^{\prime}(\sqrt{\cdot})$ ). Interested readers may consult a number of courses to obtain deeper insights into the use of robust functions and on the convergence of the associated semi-quadratic minimization process $(\mathrm{Hu}-$ ber, 1981; Geman and Reynolds, 1992; Delanay and Bresler, 1998). Such robust estimators have demonstrated significant improvements for computer vision applications (Geman and Reynolds, 1992; Mémin and Pérez, 1998). In our application, it enables us to properly deal with corrupted areas that do not fit our data model exactly.

As for matrix $\left(P^{k}\right)^{-1}$, it is directly defined from the quality index $\eta$ :

$$
\left(P^{k}\right)^{-1}(\mathbf{s}, \mathbf{s})=\left\{\begin{array}{lll}
0 & \text { if } \eta(\mathbf{s})=0, & \mathbf{s}=\mathbf{s}^{\prime} \text { and } \mathbf{s} \in C^{k} \\
1 & \text { if } \eta(\mathbf{s})=1, & \mathbf{s}=\mathbf{s}^{\prime} \text { and } \mathbf{s} \in C^{k} \\
0.5 & \text { if } \eta(\mathbf{s})=2, & \mathbf{s}=\mathbf{s}^{\prime} \text { and } \mathbf{s} \in C^{k} \\
0 & \text { else. }
\end{array}\right.
$$

In this way, the impact of the measurements in areas associated with a poor quality of the pressure level is lowered.

4.5.2 Initial conditions As no guarantee of convergence towards a global minimum can be ensured for such a nonconvex functional, the quality of results depends on the initial conditions given to the system. We chose to initialize the velocities $\tilde{\mathbf{v}}_{0}^{k}$ and the associated divergence and vorticity $\left[D_{0}^{k}, \xi_{0}^{k}\right]$ by filtering wind fields provided by the optic-flow algorithm dedicated to atmospheric layers proposed in (Héas et al., 2007), with the Gaussian Kernel $K_{\delta_{x}}$. Two inverse diagonal error covariance matrices $\left(B_{D}^{k}\right)^{-1}$ and $\left(B_{\xi}^{k}\right)^{-1}$ are defined as :

$$
\left\{\begin{array}{l}
\left(B_{D}^{k}\right)^{-1}(\mathbf{s}, \mathbf{s})=\alpha_{D} \\
\left(B_{\xi}^{k}\right)^{-1}(\mathbf{s}, \mathbf{s})=\alpha_{\zeta}
\end{array}\right.
$$

where $\alpha_{D}$ and $\alpha_{\xi}$ are fixed parameters defining the inverse of the initial variable error covariances.

4.5.3 Dynamics A nine-diagonal inverse error covariance matrix $\left(Q^{k}\right)^{-1}$ fixing the model inaccuracy with a given parameter $\alpha_{Q}$ has been defined for the dynamic model in a similar way as for the initial conditions.

All the components needed for the definition of our assimilation process have now been successively defined in sections $4.1,4.2,4.3,4.4$ and 4.5. The next section presents some experiments using synthetic and real data. 


\section{Experiments}

For a quantitative evaluation of the proposed image assimilation scheme, we have first performed experiments on synthetic image observations generated by short time numerical simulation of atmospheric layer motion. This evaluation is described in section 5.1. As the dynamic model of our assimilation process in (32) is a simplified version of the primitive shallow-water model presented in (17), two experiments related to different synthetic observations have been carried out. The first synthetic expeiment (section 5.1.1) uses images generated with the simplified model whereas the images of the second one (section 5.1.2) have been produced following the shallow-water model. Section 5.2 is devoted to the application of the assimilation scheme on real Meteosat data. The core of section 5.3 is a discussion of practical aspects.

\subsection{Synthetic image sequences}

For the sake of clarity, we denote the proposed simplified dynamic model in (32) as model- $a$ and the primitive shallow-water model (17) as model-b. For both cases, we selected realistic initial conditions (for images and motion fields) to integrate the models in an equivalent time period of $2 \mathrm{~h} 30 \mathrm{~min}$ and to create two different sequence benchmarks composed of 10 frames each, which are denoted respectively as seq- $a$ (for model- $a$ ) and seq- $b$ (for model- $b$ ). Each image is composed of $128 \times 128$ pixels and represents a spatial area of approximately $400 \mathrm{~km}^{2}$. These benchmarks have then been deteriorated by different noises and by masking operations corresponding to real situations (the masking area represents approximately $80 \%$ of the global area). We formed 4 synthetic sequences $a_{1}, a_{2}, a_{3}, a_{4}$ related to seq-a, and 4 other synthetic sequences $b_{1}, b_{2}, b_{3}, b_{4}$ related to $s e q-b$. Sequences $a_{1}, b_{1}$ and $a_{2}, b_{2}$ were composed of dense observations of $h_{o b s}^{k}$ in $\mathrm{hPa}$, corrupted by Gaussian noises with standard deviation respectively equal to $20 \%$ and $40 \%$ of the pressure amplitude. A realistic cloud classification sequence was employed to extract regions from $a_{1}, a_{2}, b_{1}$ and $b_{2}$ in order to create the noisy and incomplete synthetic sequences $a_{3}, a_{4}, b_{3}$ and $b_{4}$. To initialize the assimilation systems, we have used the synthetic ground truth values of $\tilde{\mathbf{v}}^{k}\left(t_{0}\right)$ deteriorated by Gaussian noises with standard deviation equal to $20 \%$ and $40 \%$ of the variable amplitudes respectively for experiments using synthetic sequences $a_{1}, a_{3}, b_{1}, b_{3}$ and $a_{2}, a_{4}, b_{2}, b_{4}$.

Table 1 summarizes the characteristics of the two sequences used. This table also presents the initial value of Root Mean Square Error (RMSE) of state variables. These values correspond to the discrepancy between the ground truth and the state variables obtained after a first direct integration from the noisy initial conditions. Figure 2 displays examples of noisy and sparse pressure difference observations and initial values of the state variables used in the experiments.

5.1.1 Synthetic sequence generated from the proposed simplified model In this experiment, the dynamic model involved in the generation of the image sequence exactly corresponds to the dynamics used in the assimilation process (model-a, eq. (32)). The goal of this experiment is to analyze the robustness of the assimilation with respect to noisy data and initial settings.

Comparing Table 1 and Table 2, one can note a significant decrease induced by the assimilation system on the RMSE between the actual and the estimated velocities; the final RMSE is reduced roughly by a factor of $\approx 85 \%$ by the assimilation process. Moreover, the stable behavior of the RMSE (in the four cases considered) demonstrates the robustness of the approach to cope with incomplete and noisy observations. A motion field and the associated vorticity and divergence maps obtained for experiment $a_{4}$ are plotted in the top part of figure 3 and compared to the actual solution. A comparison between those images and the images of their initial values, displayed in Figure 2, clearly illustrates the estimation efficiency.

5.1.2 Synthetic sequence generated from the primitive shallow-water model In this application, the dynamic model used for the construction of images corresponds to the primitive shallow-water model (eq (17)). Therefore, it differs from the dynamic model considered in the assimilation process. The goal of this experiment is then twofold : 1- to analyze the ability of the method to deal with an imperfect dynamic modeling involving additional uncertainty variables and 2- to evaluate the accuracy of the simplification considered in section 4.1.2.

Comparing Table 1 and Table 2, a significant decrease of the RMSE after assimilation can still be observed. The final RMSE of this experiment is reduced by approximately $75 \%$. The residual errors of the divergence and vorticity recovered are slightly above the errors obtained in the previous experiment but remains very weak. For both experiments, the estimated velocity fields are of the same order of accuracy. This result demonstrates the capacity of the simplified dynamics together with a weak constraint assimilation formulation to fit an exact shallow-water dynamic. Here again, the stable behavior of the RMSE on vorticity, divergence and motion fields for the four experimental cases is noticeable. Vorticity, divergence and motion field estimates obtained from experiment $b_{2}$ are presented at the bottom of Figure 3. The efficiency of the process can be observed by comparing those images with the images displayed in Figure 2 and corresponding to the initial values of the state variables for the same benchmark.

The performance of the simplified dynamic model has been assessed from two different synthetic cases. These experiments have shown the capacity of the proposed assimilation scheme to estimate accurate motion fields from synthetic pressure sequences. Let us now study the behavior of the approach on a real case.

\subsection{METEOSAT Satellite image sequence}

We turn now to a qualitative evaluation of our method on METEOSAT Second Generation (MSG) meteorological image sequences acquired over the Northern Atlantic Ocean on 5 June 2004 from $13 \mathrm{H} 30$ until $15 \mathrm{H} 45$ UTC at the rate of one image per 15 minutes. This benchmark data, which 
has been provided by the EUMETSAT consortium, is composed of 10 frames sequence of top of cloud pressure and cloud-classification images. The image spatial resolution is $3 \times 3 \mathrm{~km}^{2}$ at the center of the whole Earth image disk. The cloud-classifications were used to segment images into $K=3$ broad layers, at low, intermediate and high altitude ${ }^{6}$. Applying the methodology described in section 4.4, pressure difference images for the 3 layers were derived from pressure images.

In Figure 4 we present a sample of the motion fields estimated for two different layers together with the original observations. The motion fields estimated for the different layers are consistent with a visual inspection of the sequence. In particular, several motion differences between layers are very relevant. For instance, near the bottom left corner of the images, the lower layer possesses a southward motion while the intermediate layer moves northward.

In addition, let us outline that the motion of some small cloud structures have been well characterized by this assimilation scheme. This is due to the fact that the shallow-water assumption is not assumed to be strictly respected everywhere. Thus, small scale information from the image observations may locally generate a motion field which significantly departs from the shallow-water solution. This constitutes a benefit of our approach since our preliminary experiments in (Corpetti et al., 2008) have shown that the motions of small structures can not be extracted from an assimilation scheme combining a primitive shallow-water model (17) and pressure image measurements. Of course, at this stage, the question of the validation is crucial and at the moment, no ground truth is available (especially concerning the small scales structures). One possible and interesting idea would be to compare our estimation with AMV products. However, this would be difficult as it would require the development of adapted procedures to deal with the resulting sparsity of AMV data when one focuses on a particular layer. The motion vectors from AMV are indeed assigned to a precise height and the way to compare this kind of data with our motion fields is not trivial. This issue will be the object of a future study. Nevertheless, we have compared our estimations with the velocity fields obtained by the layer-dedicated optic-flow method of (Héas et al., 2007) and whose results have been validated using several Meteosat data. The maps of divergence and vorticity corresponding to this "reference" estimator are displayed in Figure 5. It can be observed, from a visual comparison between the lower layer vorticitydivergence components estimated by (Héas et al., 2007) and by the proposed assimilation system, that our estimations are in accordance with these motion fields. Indeed, timepersistent large structures of the flow are accurately estimated by both methods while noise and time-inconsistent structures have only been removed by the assimilation approach.

\footnotetext{
6 The number of $K=3$ layers is due to the nature of the input data-based on routines from Eumetsat- but any number of levels is supported by the approach.
}

\subsection{Discussion on experiments}

5.3.1 Comments This experimental part has been divided into two subsections using synthetic and real data. The two experiments with synthetic data have illustrated the accuracy and the stability of our assimilation system in presence of noisy and sparse observations. The second set of experiments allowed us to assess the validity of the simplified divergence vorticity form of the shallow-water model considered in this study.

For the experiments on real situations we have checked that our scheme supplied motion fields that are visually plausible and in accordance with a dedicated atmospheric layer motion estimator. It is obvious that the validation has to be more complete. A complete evaluation was beyond the scope of this study. In the near future we plan to compare thoroughly our results with AMV data and in situ data provided by dedicated probes (radiosondes for instance) to obtain reference velocities at some points and to allow a precise statistical assessment in the vicinity of these points. Another key point of error analysis relies on the study of the adjoint variables $\lambda$ defined in relation (22) when the process has converged. These components are indeed related to the errors between the dynamic model and the observations and are likely to exhibit some interesting properties and information. This point will be also the object of future studies.

5.3.2 Computation time The execution time for one forward-backward computation depends on $i$ ) the size of images ii) the number of images and iii) the magnitude of the vorticity and divergence components: as mentioned in section 4.3, an explicit Runge-Kutta scheme has indeed been used for the temporal (forward and backward) integration (Kurganov and Tadmor, 2000). To ensure stability of this scheme, the time-step discretization is inversely proportional to the magnitude of the velocity. The initialization plays also a key role in the computational cost since the iterative process converges faster if one correctly initializes the system state.

As an example, in our application, the estimation of the complete system state takes approximately $1 \mathrm{~min}$ for the synthetic sequences $(128 \times 128$ pixels $)$ whereas the estimation on a real layer $(1024 \times 1024$ pixels $)$ takes around $30 \mathrm{~min}$ for a Matlab code, on a PC $2.33 \mathrm{~Hz}$ with $3 \mathrm{Go}$ of RAM. An efficient "C" implementation would obviously significantly reduce the computational time.

5.3.3 Parameter values The numerical values of the different parameters are set according to the range of variation of the quantity of interest. The values used in all our applications are given in Table 3.

\section{Conclusion}

In this paper, we have proposed an assimilation approach enabling, for the first time, a dynamically consistent estimation of a sequence of dense and layered atmospheric wind fields from entire satellite image sequences.

The motion estimator is applied to sparse pressure 
difference images corresponding to a stack of layers in a stratified atmosphere. A method was proposed to obtain such images from top of cloud pressure images and classification, which are routinely supplied by the EUMETSAT consortium.

The dynamic constraint of our system suggested relying on the shallow-water assumption. We have shown that a perfect shallow-water modeling approach is likely to be unstable for the Meteosat data considered. We have then derived a simplified dynamic modeling with uncertainty terms. This model corresponds to a simplification of the vorticity divergence form of a shallow-water dynamics for which the momentum equations are independent of the layer thickness. The mass conservation law complements these dynamics and provides the basis of an original image-adapted observation operator.

An evaluation performed on synthetic image sequences and on real METEOSAT top of cloud pressure image sequences has shown that the technique proposed enables characterizing time-consistent wind fields at large and small scales.

\section{APPENDIX A: Scale analysis of filtered hydrostatic primitive equations}

For mesoscale atmospheric motions at horizontal scale of $100 \mathrm{~km}$, the following scale characteristics hold :

$\begin{array}{cccc}U & \sim & 10 \mathrm{~ms}^{-1} & \text { horizontal velocity } \\ W & \sim & 1 \mathrm{~ms}^{-1} & \text { vertical velocity } \\ L & \sim & 10^{5} \mathrm{~m} & \text { length } \\ H & \sim & 10^{3} \mathrm{~m} & \text { depth } \\ \delta p & \sim & 10^{3} \mathrm{~Pa} & \text { horizontal pressure variation } \\ \rho & \sim & 1 \mathrm{~kg} \mathrm{~m}^{-3} & \text { density } \\ \Delta t & \sim & 10^{3} \mathrm{~s} & \text { temporal sampling period } \\ \nu & \sim 10^{-5} \mathrm{~m}^{2} \mathrm{~s}^{-1} & \text { friction coefficient } \\ a & \sim & 10^{6} \mathrm{~m} & \text { Earth radius } \\ \Omega \sin \phi & \sim 10^{-4} \mathrm{~s}^{-1} & \text { Coriolis coefficient } \\ \frac{U}{2 \Omega \sin \phi L} & \sim & 0.5 & \text { Rossby number }\end{array}$

The horizontal primitive hydrostatic equations of relation (2) are :

$$
\left\{\begin{array}{l}
\underbrace{\frac{d u}{d t}}-\underbrace{\frac{u v \tan \phi}{a}}+\underbrace{\frac{u w}{a}}+\underbrace{\frac{p_{x}}{\rho}}=+2 \Omega v \sin \phi-2 \Omega w \cos \phi+\mathcal{F}_{u} \\
\underbrace{\frac{d v}{d t}}_{A}+\underbrace{\frac{u^{2} \tan \phi}{a}}_{B}+\underbrace{\frac{v w}{a}}_{C}+\underbrace{\frac{p_{y}}{\rho}}_{D}=-\underbrace{2 \Omega u \sin \phi}_{E} \underbrace{}_{F}+\underbrace{\mathcal{F}_{v}}_{G}
\end{array}\right.
$$

An analysis of these terms using the characteristic scales is given in Table 4.

We conclude that terms B, C, F and G can be ignored in comparison to the other terms. Moreover it is well known that, for high Reynolds numbers characteristic of atmospheric flows, due to the energy dissipation at unobservable scales, turbulent viscosity dissipation $\mathcal{T}$ can not be ignored in Navier-Stokes numerical simulations (Meneveau and Katz, 2000; Sagaut, 1998; Smagorinsky, 1963). We thus obtain the filtered momentum equations written in a vectorial form:

$$
\rho \frac{d \tilde{\mathbf{v}}}{d t}=-\nabla \tilde{p}-2 \Omega \sin \phi\left[\begin{array}{cc}
0 & -1 \\
1 & 0
\end{array}\right] \tilde{\mathbf{v}}+\mathcal{T} .
$$

\section{APPENDIX B: Calculation of layer average densities}

The integration of the hydrostatic relation between altitudes $z_{0}$ and $z$ (or pressure $p_{0}$ and $p$ ), with a constant lapse rate may be written as:

$$
\int_{p_{0}}^{p} \frac{d p^{\prime}}{p^{\prime}}=-\int_{z_{0}}^{z} \frac{g}{R\left(T_{0}+\gamma z^{\prime}\right)} d z^{\prime}
$$

This expression gives rise after some calculation to the expression of density as a function of pressure (Holton, 1994)):

$$
\rho(p)=\frac{p_{0}}{R T_{0}}\left(\frac{p}{p_{0}}\right)^{\frac{\gamma R}{g}+1} .
$$

Computing the vertical density average, the mean density related to the $k$-th layer reads :

$$
\begin{aligned}
\rho_{0}^{k} & =\frac{1}{\bar{p}^{k+1}-\bar{p}^{k}} \int_{\bar{p}^{k}}^{\bar{p}^{k+1}} \rho(p) d p \\
& =\frac{p_{0}^{2}}{\left(\bar{p}^{k+1}-\bar{p}^{k}\right)\left(\frac{\gamma R}{g}+2\right) R T_{0}}\left[\left(\frac{p}{p_{0}}\right)^{\frac{\gamma R}{g}+2}\right]_{\bar{p}^{k}}^{\bar{p}^{k+1}} .
\end{aligned}
$$

Note that a constant lapse rate $\gamma$, that is to say a linear variation of temperature with altitude, is a rough approximation in the troposphere. However, as we are averaging the obtained density law vertically and horizontally over the whole layer spatial support, such an approximation should have a minor impact on the accuracy of the modeling.

\section{APPENDIX C: Scale analysis of Saint Venant's model with pressure image observations}

For mesoscale atmospheric motions observed through a METEOSAT images sequence which has been low-pass filtered to obtain a characteristic horizontal scale of $100 \mathrm{~km}$, the following characteristic scales hold (with $: h^{k}=p^{k}-p^{k+1}$ )

$$
\begin{array}{lccc}
\mathbf{v} & \sim 10 \mathrm{~ms}^{-1} & \text { horizontal velocity } \\
h^{k} & \sim 10^{4} \mathrm{~Pa} & \text { pressure differences } \\
\rho & \sim 1 \mathrm{~kg} \mathrm{~m}^{-3} & \text { density } \\
\Delta_{t} & \sim 10^{3} \mathrm{~s} & \text { temporal sampling period } \\
\Delta_{x y} & \sim 10^{5} \mathrm{~m} & \text { spatial scale } \\
f^{\phi} & \sim 10^{-3} \mathrm{~s}^{-1} & \text { Coriolis coefficient } \\
\nu_{\mathcal{T}} & \sim 10^{4} \mathrm{~m}^{2} \mathrm{~s}^{-1} & \text { turbulent diffusion (cf (5)) }
\end{array}
$$

The system to study reads:

$$
\begin{aligned}
& \underbrace{\frac{\partial\left(\tilde{\mathbf{q}}^{k}\right)}{\partial t}}_{A}+\underbrace{\operatorname{div}\left(\frac{1}{\tilde{h}^{k}} \tilde{\mathbf{q}}^{k} \otimes \tilde{\mathbf{q}}^{k}\right)}_{B}+\underbrace{\frac{1}{2 \rho_{0}^{k}} \nabla_{x y}\left(\tilde{h}^{k}\right)^{2}}_{C}+\underbrace{\left[\begin{array}{cc}
0 & -1 \\
1 & 0
\end{array}\right] f^{\phi} \tilde{\mathbf{q}}^{k}}_{D} \\
& =\underbrace{\nu_{\mathcal{T}}^{k} \Delta\left(\tilde{\mathbf{q}}^{k}\right)}_{E},
\end{aligned}
$$

where $\left(\nu_{\mathcal{T}}=\left(C \delta_{x}\right)^{2} \sqrt{2\left(\tilde{u}_{x}^{2}+\tilde{v}_{y}^{2}+\left(\tilde{u}_{y}+\tilde{v}_{x}\right)^{2}\right)}\right)$ (cf relation (5)). Noting that the Pascal unit is $h P a=k g \cdot \mathrm{s}^{-2} \cdot \mathrm{m}^{-1}$ 
and recalling that $\mathbf{q}=h^{k} \mathbf{v}$ ), a scale analysis of this system is given in Table 5 .

This corresponds to the order of magnitudes of the quantities of interest under the shallow-water approximation. From Table 5, we observe that term $\mathrm{C}$ of eq $\mathrm{C} 1$ is slightly higher than all the others. In practice, as soon as the shallow-water assumption breaks, the pressure difference of a layer $\tilde{h}^{k}$ grows and we observed that the term $\left(\nabla_{x y}\left(\tilde{h}^{k}\right)^{2}\right)$ can reach the magnitude of $10^{7} \mathrm{~kg} \cdot \mathrm{s}^{-4}$. The sensitivity of this term w.r.t the assumption generates strong numerical instabilities. This primitive relation is therefore not adapted to the assimilation of sparse images of pressure differences when one wants to deal with corrupted input data.

\section{REFERENCES}

Andersson, E., J. Pailleux, J.-N. Thépaut, J. R. Eyre, A. P. McNally, G. A. Kelly, and P. Courtier, 1994: Use of cloudcleared radiances in three/four-dimensional variational data assimilation. Q. J. R. Meteorol. Soc., 120, 627-653.

Bayler, G., R. M. Aune, and W. H. Raymond, 2000: NWP cloud initialization using GOES sounder data and improved modeling of nonprecipitating clouds. Monthly Weather Review, 128(11), 3911-3920.

Benjamin, S., D. Kim, and J. Brown, 2002: Cloud/hydrometeor initialization in the 20-km RUC using GOES and radar data. In proceedings of 10th Conf. on Aviation, Range, and Aerospace Meteorology, Amer. Meteor. Soc., Portland, Orlando, USA.

Bennet, A., 1992: Inverse Methods in Physical Oceanography. Cambridge University Press.

Bennet, A., B. Chua, and L. Leslie, 1996: Generalized inversion of a global numerical weather prediction model. Meteor. Atmos. Phys., 60, 165-178.

Bennett, A. and M. Thorburn, 1992: The generalized inverse of a nonlinear quasigeotrophic ocean circulation model. J. Phys. Ocean., 22, 213-230.

Corpetti, T., P. Héas, E. Mémin, and N. Papadakis, 2008: Pressure image assimilation for atmospheric motion estimation. Research Report 6507, INRIA

Corpetti, T., D. Heitz, G. Arroyo, E. Mémin, and A. SantaCruz, 2006: Fluid experimental flow estimation based on an optical-flow scheme. Experiments in fluids, 40, 80-97.

Corpetti, T., E. Mémin, and P. Pérez, 2002: Dense estimation of fluid flows. IEEE Trans. Pattern Anal. Machine Intell., 24(3), 365-380.

Courtier, P. and O. Talagrand, 1990: Variational assimilation of meteorological observations with the direct and adjoint shallow-water equations. Tellus, 42A, 531-549.

Courtier, P., J.-N. Thépaut, and A. Hollingsworth, 1994: A strategy for operational implementation of $4 \mathrm{D}-\mathrm{VAR}$, using an incremental approach. Q. J. R. Meteorol. Soc., 120, 13671388.

Cuzol, A., P. Hellier, and E. Mémin, 2007: A low dimensional fluid motion estimator. Int. Journ. on Computer Vision, 75(3), 329-349.

Cuzol, A. and E. Mémin, 2008: A stochastic filter technique for fluid flows velocity fields tracking. IEEE Trans. Pattern Anal. Machine Intell. in press.

D'Adamo, J., N. Papadakis, E. Mémin, and G. Artana, 2007: Variational assimilation of pod low-order dynamical systems. Journal of Turbulence, 8(9), 1-22.

De Saint-Venant, A., 1871: Théorie du mouvement nonpermanent des eaux, avec application aux crues des rivières et à l'introduction des marées dans leur lit. C. R. Acad. Sc. Paris (in French), 73, 147-154.

Delanay, A. and Y. Bresler, 1998: Globally convergent edgepreserving regularized reconstruction: an application to limited-angle tomography. IEEE Trans. Image Processing, $7(2), 204-221$.

Frisch, U., 1995: Turbulence : the legacy of A.N. Kolmogorov. Cambridge university press.

Geman, D. and G. Reynolds, 1992: Constrained restoration and the recovery of discontinuities. IEEE Trans. Pattern Anal. Machine Intell., 14(3), 367-383.

Ghil, M. and P. Malanotte-Rizzoli, 1991: Data assimilation in meteorology and oceanography. Adv. Geophys., 23, 141-266.

Héas, P. and E. Mémin, 2008: 3D motion estimation of atmospheric layers from image sequences. IEEE Trans. Geoscience and Remote Sensing. in press.

Héas, P., E. Mémin, N. Papadakis, and A. Szantai, 2007: Layered estimation of atmospheric mesoscale dynamics from satellite imagery. IEEE Trans. Geoscience and Remote Sensing, 45(12), 4087-4104.

Holmlund, K., 1998: The utilization of statistical properties of satellite-derived atmospheric motion vectors to derive quality indicators. Weather and Forecasting, 13(4), 1093-1104.

Holton, J., 1994: An introduction to dynamic meteorology, $4^{\text {th }}$ edition. Academic press.

Horn, B. and B. Schunck, 1981: Determining optical flow. Artificial Intelligence, 17, 185-203

Huber, P., 1981: Robust Statistics. John Wiley \& Sons.

Kim, D. S. and S. G. Benjamin, 2000: Assimilation of cloud-top pressure derived from GOES sounder data into MAPS/RUC. In 10th Conf. on Satellite Meteorology and Oceanography, Soc., A. M., editor, Long Beach, CA, 110-113.

Kurganov, A. and E. Tadmor, 2000: New high-resolution central schemes for nonlinear conservation laws and convetiondiffusion equations. J. Comput. Phys., 160(1), 241-282.

Le Dimet, F.-X. and J. Blum, 2002: Assimilation de données pour les fluides géophysiques. MATAPLI, Bulletin de la SMAI (in French), 67, 35-55.

Le Dimet, F.-X. and O. Talagrand, 1986: Variational algorithms for analysis and assimilation of meteorological observations: theoretical aspects. Tellus, 38A, 97-110.

Leese, J., C. Novack, and B. Clark, 1971: An automated technique for obtained cloud motion from geosynchronous satellite data using cross correlation. Journal of applied meteorology, 10, $118-132$.

Lions, J., 1971: Optimal control of systems governed by PDEs. Springer-Verlag.

Lucas, B. and T. Kanade, 1981: An iterative image registration technique with an application to stereovision. In Int. Joint Conf. on Artificial Intel. (IJCAI), 674-679.

Lutz, H., 1999: Cloud processing for meteosat second generation. Technical report, European Organisation for the Exploitation of Meteorological Satellites (EUMETSAT), Available at: http://www.eumetsat.de, 26.

Mansour, N. N., J. H. Ferziger, and W. C. Reynolds, 1978: Largeeddy simulation of a turbulent mixing layer. Technical report, Report TF-11, Thermosciences Div., Dept. of Mech. Eng., Standford University.

Mémin, E. and P. Pérez, 1998: Dense estimation and objectbased segmentation of the optical flow with robust techniques. IEEE Trans. Image Processing, 7(5), 703-719.

Meneveau, C. and J. Katz, 2000: Scale-invariance and turbulence models for large-eddy simulation. Annu. Rev. Fluid Mech., $32,1: 32$.

Menzel, W. P., W. L. Smith, and T. Stewart, 1983: Improved cloud motion wind vector and altitude assignment using vas. Journal of Applied Meteorology, 22, 377-384.

Oksendal, B., 1998: Stochastic differential equations. Spinger- 
Verlag.

Papadakis, N., T. Corpetti, and E. Mémin, 2007: Dynamically consistent optical flow estimation. In proceedings of IEEE Int. Conf. Comp. Vis.(ICCV'07).

Papadakis, N. and E. Mémin, 2007: A variational method for joint tracking of curve and motion. Technical Report 6283, INRIA Research report.

Papadakis, N. and E. Mémin, 2008: A variational technique for time consistent tracking of curves and motion. Journal of Mathematical Imaging and Vision, 31(1), 81-103.

Rabier, F., J.-N. Thépaut, and P. Courtier, 1998: Extended assimilation and forecasts experiments with a four-dimensional variational assimilation system. $Q J R$ Meteorol Soc, 124, 1861-1888.

Ruhnau, P. and C. Schnörr, 2007: Optical stokes flow estimation: An imaging-based control approach. Exp.in Fluids, 42(1), $61-78$.

Sagaut, P., 1998: Introduction a la simulation des grandes echelles pour les ecoulements de fluide incompressible. Springer Verlag, Collection : Mathematiques et applications 30 (in French).

Schmetz, J., C. Geijo, W. P. Menzel, K. Strabala, L. Van De Berg, K. Holmlund, and S. Tjemkes, 1995: Satellite observations of upper tropospheric relative humidity, clouds and wind field divergence. Contributions to Atmospheric Physics, 68(4), $345-357$.

Schmetz, J., K. Holmlund, J. Hoffman, B. Strauss, B. Mason, V. Gaertner, A. Koch, and L. V. D. Berg, 1993: Operational cloud-motion winds from meteosat infrared images. Journal of Applied Meteorology, 32(7), 1206-1225.

Smagorinsky, J., 1963: General circulation experiments with the primitive equations. Monthly Weather Review, 91(3), 99164.

Talagrand, O., 1997: Assimilation of observations, an introduction. J. Meteor. Soc. Jap., 75, 191-209.

Talagrand, O. and P. Courtier, 1987: Variational assimilation of meteorological observations with the adjoint vorticity equation. I: Theory. J. of Roy. Meteo. Soc., 113, 1311-1328.

Taylor, G., 1932: The transport of vorticity and heat through fluids in turbulent motion. In Proc London Math Soc. Ser A, 151-421.

Vidard, P., E. Blayo, F.-X. Le Dimet, and A. Piacentini, 2000: $4 \mathrm{~d}$ variational data analysis with imperfect model. Flow, Turbulence and Combustion, 65(3-4), 489-504.

$\mathrm{Xu}, \mathrm{Z}$. and C.-W. Shu, 2006: Anti-diffusive finite difference weno methods for shallow water with transport of pollutant. Journal of Computational Mathematics, 24, 239-251.

Yuan, J., C. Schnoerr, and E. Mémin, 2007: Discrete orthogonal decomposition and variational fluid flow estimation. J. of Math. Imaging and Vision, 28(1), 67-80.

Zhou, L., C. Kambhamettu, and D. Goldgof, 2000: Fluid structure and motion analysis from multi-spectrum 2D cloud images sequences. In Proc. Conf. Comp. Vision Pattern Rec., volume 2, Hilton Head Island, USA, 744-751. 


\section{Figure legend}

- Caption Fig. 1: Image observations issued from METEOSAT (Northern Atlantic Ocean on June 2004 from $13 \mathrm{~h} 30$ until 15H45 UTC). From top to bottom : cloud top pressure image; classification into low (in dark gray), medium (in light gray) and high (in white) clouds; pressure difference of the higher layer; pressure difference of the intermediate layer; pressure difference of the lower layer. Black regions correspond to missing observations and white lines represent coastal contours, meridians and parallels (every $10^{\circ}$ ).

- Caption Fig. 2: Examples of variables at initial time before assimilation with images issued from model-a (top), model-b (middle) and the corresponding ground truth (bottom). For each experiment, the columns exhibit blurred initial difference pressure image $\tilde{h}^{k}$, wind $\tilde{\mathbf{v}}^{k}$, vorticity $\tilde{\zeta}^{k}$ and divergence $\tilde{D}^{k}$ respectively. The top part corresponds to the experiment $a_{4}$ whereas the middle part represents data of experiment $b_{2}$

- Caption Fig. 3: Variables after assimilation with images issued from model-a (top) and model-b (bottom). The corresponding experiments are $a_{4}$ (top) and $b_{2}$ (bottom). The first column corresponds to the wind $\tilde{\mathbf{v}}^{k}$, the second to the vorticity $\tilde{\zeta}^{k}$ and the third to the divergence $\tilde{D}^{k}$ fields at $\mathrm{t}=1 \mathrm{~h} 15 \mathrm{~min}$. For each experiment, the first line corresponds to the estimated variables whereas the second line corresponds to the ground truth.

- Caption Fig. 4: Horizontal wind fields estimated by assimilation with an imperfect modeling for the intermediate layer (top part) and the lower layer (bottom part). For each layer, the first line represents 4 images of the sequences. The three first motion fields of the second line correspond to estimation at $t=0 \mathrm{~min}$, $t=1 h 15 \min$ and $t=2 h 30$ min respectively. The last motion field is the difference $\tilde{\mathbf{v}}^{k}(2 h 30)-\tilde{\mathbf{v}}^{k}(1 h 15)$ and illustrates the temporal changes.

- Caption Fig. 5: Comparison of results from assimilation (top) and optical-flow (bottom) for the lower layer at $t=0 \mathrm{~min}, t=1 \mathrm{~h} 15 \mathrm{~min}$ and $t=2 \mathrm{~h} 30 \mathrm{~min}$ respectively. The first line represents the vorticity and the second line the divergence. One can conclude that both estimations are in accordance but the one from the assimilation has a better temporal consistency. 
Table 1. Initial setup used for assimilation with images issued from model-a (top) and model-b (bottom). Root Mean Square Error (RMSE) on the vorticity $\tilde{\zeta}^{k}$, divergence $\tilde{D}^{k}$ and wind norm $\tilde{\mathbf{v}}^{k}$ obtained at all times $t$ of the image synthetic sequences.

\begin{tabular}{cccccc} 
& Mask & $\begin{array}{c}\text { Noise } \\
\%\end{array}$ & $\begin{array}{c}\text { rmse on all } \tilde{\zeta}^{k}(t) \\
(1 / \text { frame })\end{array}$ & $\begin{array}{c}\text { rmse on all } \tilde{D}^{k}(t) \\
(1 / \text { frame })\end{array}$ & $\begin{array}{c}\text { rmse on all }\left|\tilde{\mathbf{v}}^{k}(t)\right| \\
(\text { pixel/frame })\end{array}$ \\
\hline \hline$a_{1}$ & no & 20 & 0.01119 & 0.01343 & 0.08798 \\
\hline$a_{2}$ & no & 40 & 0.01481 & 0.01518 & 0.09512 \\
\hline$a_{3}$ & yes & 20 & 0.01119 & 0.01343 & 0.08798 \\
\hline$a_{4}$ & yes & 40 & 0.01481 & 0.01518 & 0.09512 \\
\hline \hline$b_{1}$ & no & 20 & 0.01297 & 0.00932 & 0.09352 \\
\hline$b_{2}$ & no & 40 & 0.01670 & 0.01814 & 0.1009 \\
\hline$b_{3}$ & yes & 20 & 0.01297 & 0.00932 & 0.09352 \\
\hline$b_{4}$ & yes & 40 & 0.01670 & 0.01814 & 0.1009 \\
\hline
\end{tabular}

Table 2. Final estimation errors obtained by our assimilation approach with images issued from model-a (top) and model-b (bottom). Root Mean Square Error (RMSE) and residual error (RE) on estimates $\tilde{\zeta}^{k}, \tilde{D}^{k}$ and $\tilde{\mathbf{v}}^{k}$ obtained at all times $t$ of the image synthetic sequences.

\begin{tabular}{ccccccc} 
& Errors on $\tilde{\zeta}^{k}(t)$ & \multicolumn{2}{c}{ Errors on $\tilde{D}^{k}(t)$} & \multicolumn{2}{c}{ Errors on $\left|\tilde{\mathbf{v}}^{k}(t)\right|$} \\
& $\begin{array}{c}\text { RMSE } \\
(1 / \text { frame })\end{array}$ & $\begin{array}{c}\text { RE } \\
\%\end{array}$ & $\begin{array}{c}\text { RMSE } \\
(1 / \text { frame) }\end{array}$ & $\begin{array}{c}\text { RE } \\
\%\end{array}$ & $\begin{array}{c}\text { RMSE } \\
\text { (pixel/frame) }\end{array}$ & $\begin{array}{c}\text { RE } \\
\%\end{array}$ \\
\hline \hline$a_{1}$ & 0.00379 & 8.71 & 0.00182 & 9.99 & 0.01787 & 9.59 \\
\hline$a_{2}$ & 0.00405 & 8.91 & 0.00214 & 10.87 & 0.01783 & 11.12 \\
\hline$a_{3}$ & 0.00378 & 9.20 & 0.00182 & 11.61 & 0.01782 & 9.58 \\
\hline$a_{4}$ & 0.00405 & 9.51 & 0.00214 & 11.74 & 0.01784 & 11.11 \\
\hline \hline$b_{1}$ & 0.00514 & 8.94 & 0.00444 & 13.21 & 0.01883 & 9.77 \\
\hline$b_{2}$ & 0.00536 & 9.37 & 0.00450 & 13.62 & 0.02139 & 11.27 \\
\hline$b_{3}$ & 0.00514 & 9.43 & 0.00441 & 13.41 & 0.02000 & 10.61 \\
\hline$b_{4}$ & 0.00560 & 10.69 & 0.00481 & 14.74 & 0.02429 & 13.45 \\
\hline
\end{tabular}

Table 3. Default parameters: values of the covariance parameters used in our applications

\begin{tabular}{ccc} 
Variable & Value & Description \\
\hline \hline$\alpha_{\text {obs }}$ & 2 & Error cov. observation (eq (43)) \\
$\sigma$ & 5 & Outlier parameter (eq (43)) \\
$\alpha_{D}$ & $10^{-3}$ & Error cov. on initial $D$ (eq (45)) \\
$\alpha_{\zeta}$ & $10^{-3}$ & Error cov. on initial $\zeta$ (eq (45)) \\
$\alpha_{Q}$ & $10^{-3}$ & Error cov. on dynamics (section 4.5.3)
\end{tabular}

Table 4. Scale analysis of the horizontal primitive hydrostatic equations (A1).

\begin{tabular}{cccccccc} 
& $\mathrm{A}$ & $\mathrm{B}$ & $\mathrm{C}$ & $\mathrm{D}$ & $\mathrm{E}$ & $\mathrm{F}$ & $\mathrm{G}$ \\
\hline & & & & & & & \\
x-eq. & $\frac{d u}{d t}$ & $\frac{u v \tan \phi}{a}$ & $\frac{u w}{a}$ & $\frac{1}{\rho} \frac{\partial p}{\partial x}$ & $2 \Omega v \sin \phi$ & $2 \Omega w \cos \phi$ & $\mathcal{F}_{u}$ \\
y-eq. & $\frac{d v}{d t}$ & $\frac{u^{2} \tan \phi}{a}$ & $\frac{v w}{a}$ & $\frac{1}{\rho} \frac{\partial p}{\partial y}$ & $2 \Omega u \sin \phi$ & - & $\mathcal{F}_{v}$ \\
scales & $\frac{U}{\Delta t}$ & $\frac{U^{2}}{a}$ & $\frac{U W}{a}$ & $\frac{\delta p}{\rho L}$ & $\Omega \sin \phi U$ & $\Omega \cos \phi W$ & $\frac{\nu U}{H^{2}}$ \\
$\left(m s^{-2}\right)$ & $10^{-2}$ & $10^{-4}$ & $10^{-5}$ & $10^{-2}$ & $10^{-3}$ & $10^{-4}$ & $10^{-10}$ \\
\hline
\end{tabular}


Table 5. Scale analysis of the Saint Venant's model (C1).

$$
\text { A }
$$

B

C

D

$\mathrm{E}$

\begin{tabular}{cccccc} 
& & & & \\
x-eq. & $\frac{\partial \tilde{h}^{k} \tilde{u}^{k}}{\partial t}$ & $\frac{\partial\left(\tilde{h}^{k}\left(\tilde{u}^{k}\right)^{2}\right)}{\partial x}+\frac{\partial\left(\tilde{h}^{k} \tilde{u}^{k} \tilde{v}^{k}\right)}{\partial y}$ & $\frac{\partial\left(\tilde{h}^{k}\right)^{2}}{2 \rho_{0}^{k} \partial x}$ & $-f^{\phi} h^{k} v$ & $\nu_{\mathcal{T}}^{k}\left(\left(\tilde{h}^{k} u\right)_{x x}+\left(\tilde{h}^{k} u\right)_{y y}\right)$ \\
y-eq. & $\frac{\partial \tilde{h}^{k} \tilde{v}^{k}}{\partial t}$ & $\frac{\partial\left(\tilde{h}^{k} \tilde{u}^{k} \tilde{v}^{k}\right)}{\partial x}+\frac{\partial\left(\tilde{h}^{k}\left(\tilde{v}^{k}\right)^{2}\right)}{\partial y}$ & $\frac{\partial\left(\tilde{h}^{k}\right)^{2}}{2 \rho_{0}^{k} \partial y}$ & $f^{\phi} h^{k} u$ & $\nu_{\mathcal{T}}^{k}\left(\left(\tilde{h}^{k} v\right)_{x x}+\left(\tilde{h}^{k} v\right)_{y y}\right)$ \\
scales & $\mathbf{q} / \Delta t$ & $h^{k} \mathbf{v}^{2} / \Delta_{x y}$ & $\left(h^{k}\right)^{2} / \rho \Delta_{x y}$ & $f^{\phi} h^{k} \mathbf{v}$ & $\nu_{\mathcal{T}} h^{k} \mathbf{v} / \Delta_{x y}^{2}$ \\
$\left(k g . s^{-4}\right)$ & $10^{2}$ & $10^{1}$ & $10^{3}$ & $10^{2}$ & $10^{2}$ \\
\hline
\end{tabular}
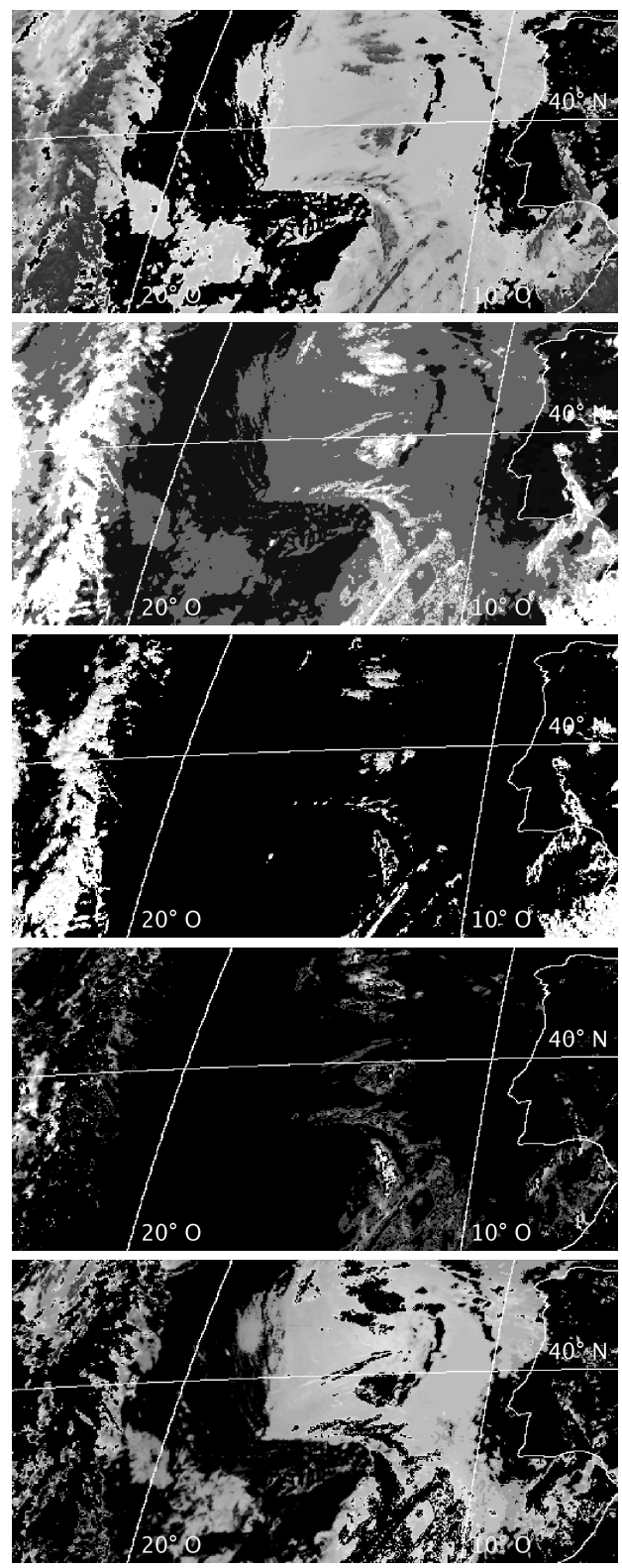

Fig. 1. Image observations issued from METEOSAT (Northern Atlantic Ocean on June 2004 from 13 h30 until 15H45 UTC). From top to bottom : cloud top pressure image; classification into low (in dark gray), medium (in light gray) and high (in white) clouds; pressure difference of the higher layer; pressure difference of the intermediate layer; pressure difference of the lower layer. Black regions correspond to missing observations and white lines represent coastal contours, meridians and parallels (every $10^{\circ}$ ). 

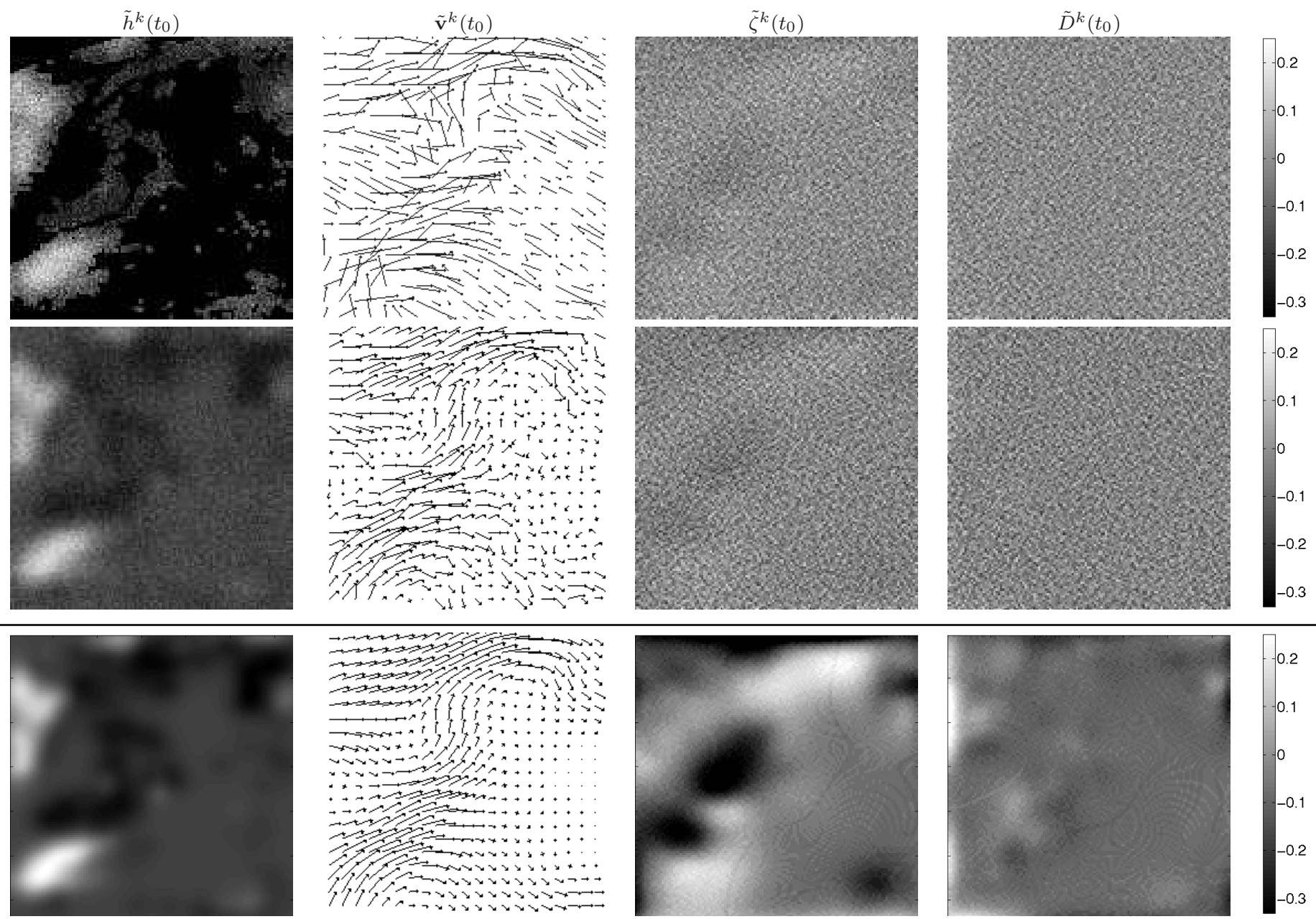

Fig. 2. Examples of variables at initial time before assimilation with images issued from model-a (top), model-b (middle) and the corresponding ground truth (bottom). For each experiment, the columns exhibit blurred initial difference pressure image $\tilde{h}^{k}$, wind $\tilde{\mathbf{v}}^{k}$, vorticity $\tilde{\zeta}^{k}$ and divergence $\tilde{D}^{k}$ respectively. The top part corresponds to the experiment $a_{4}$ whereas the middle part represents data of experiment $b_{2}$ 


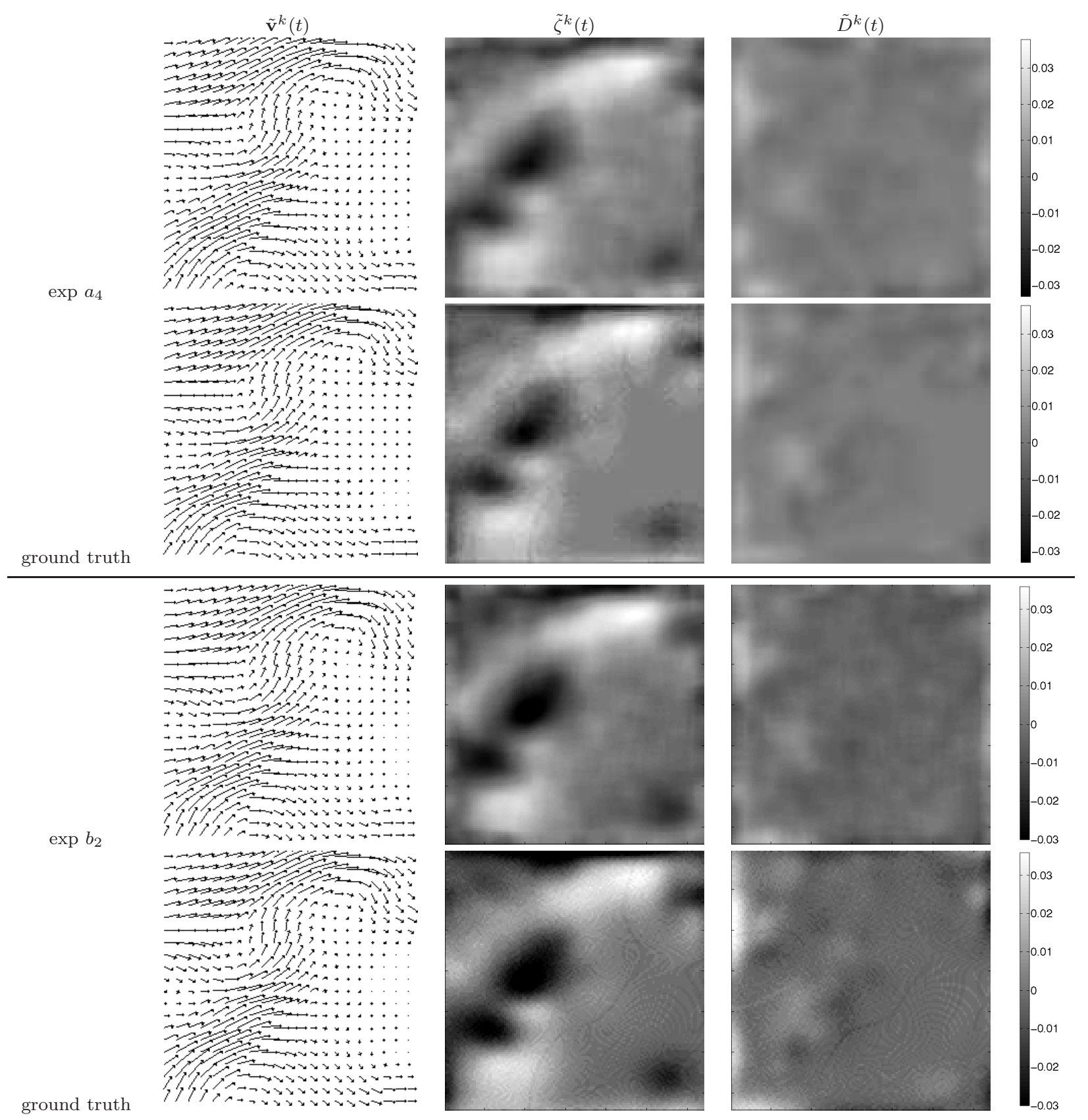

Fig. 3. Variables after assimilation with images issued from model-a (top) and model- $b$ (bottom). The corresponding experiments are $a_{4}$ (top) and $b_{2}$ (bottom). The first column corresponds to the wind $\tilde{\mathbf{v}}^{k}$, the second to the vorticity $\tilde{\zeta}^{k}$ and the third to the divergence $\tilde{D}^{k}$ fields at $\mathrm{t}=1 \mathrm{~h} 15 \mathrm{~min}$. For each experiment, the first line corresponds to the estimated variables whereas the second line corresponds to the ground truth. 

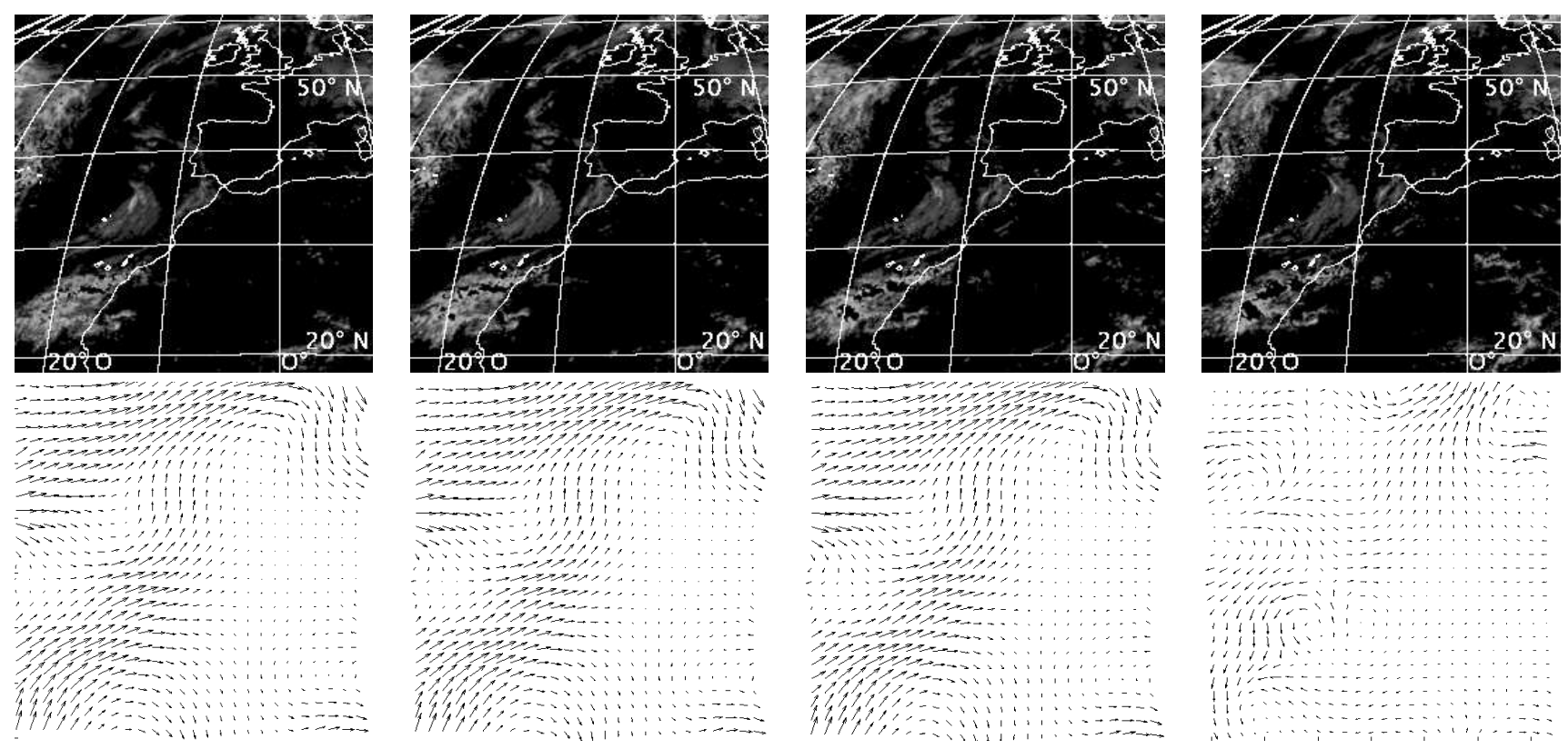

$\tilde{\mathbf{v}}^{k}(0 \min )$
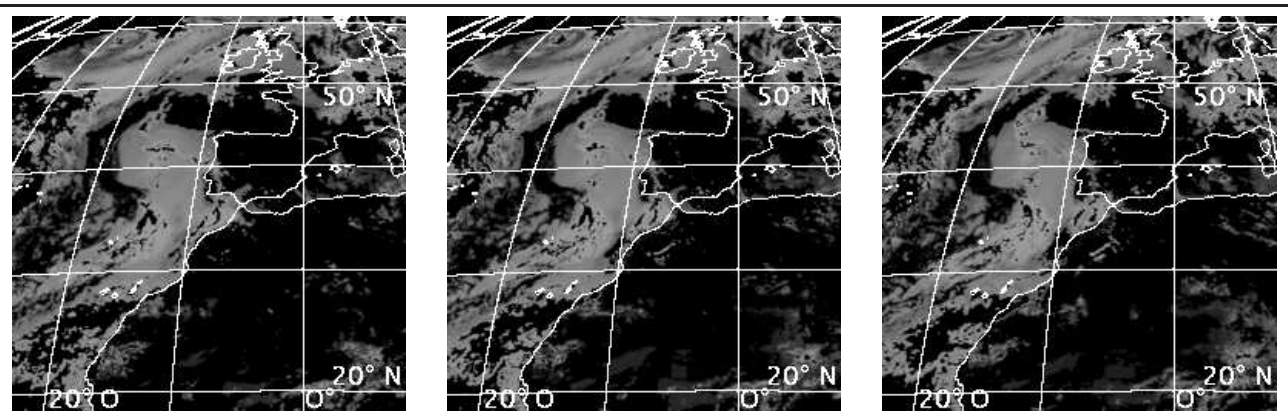

$\tilde{\mathbf{v}}^{k}(2 h 30)-\tilde{\mathbf{v}}^{k}(1 h 15)$
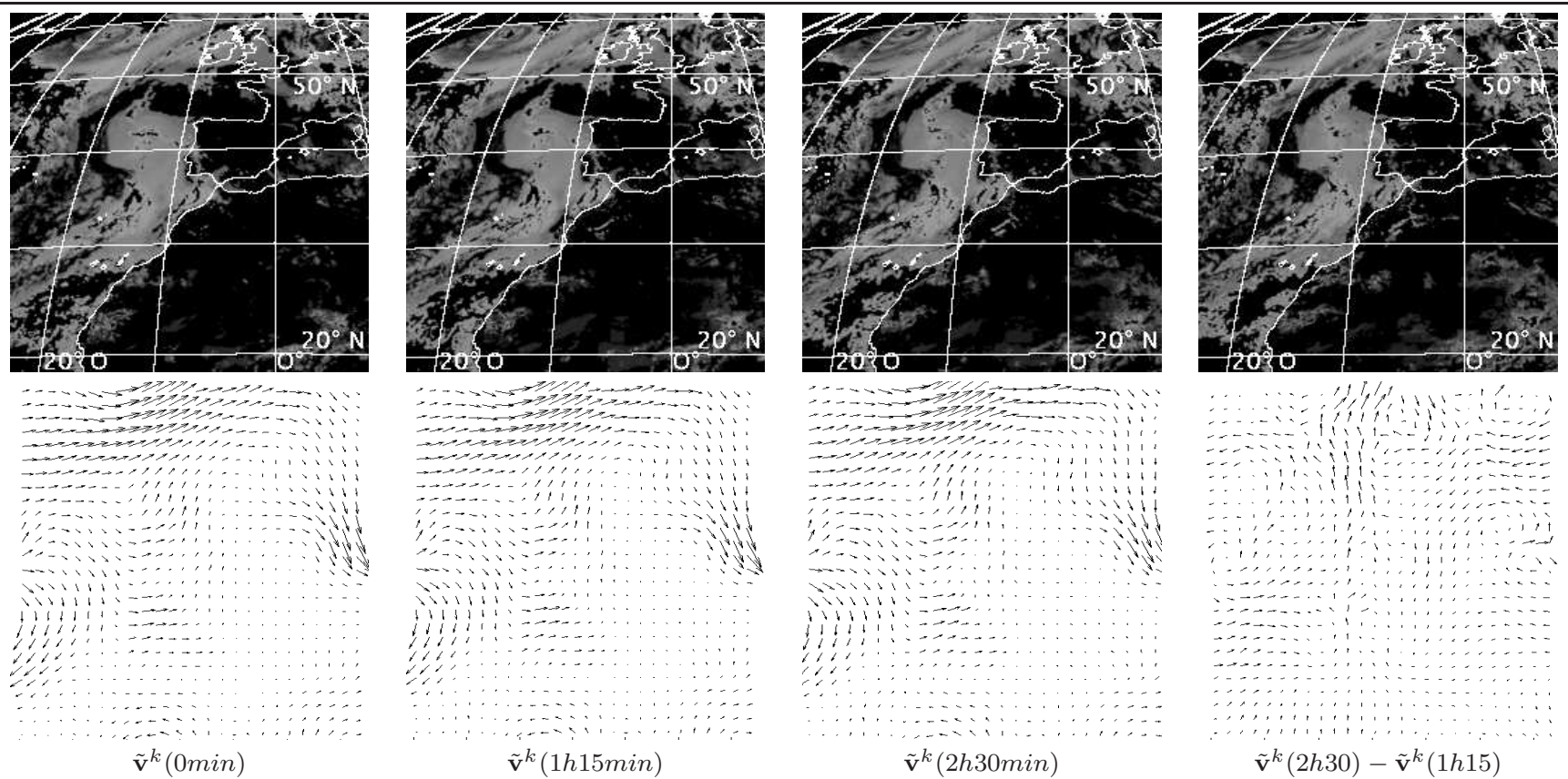

Fig. 4. Horizontal wind fields estimated by assimilation with an imperfect modeling for the intermediate layer (top part) and the lower layer (bottom part). For each layer, the first line represents 4 images of the sequences. The three first motion fields of the second line correspond to estimation at $t=0 \mathrm{~min}, t=1 \mathrm{~h} 15 \mathrm{~min}$ and $t=2 \mathrm{~h} 30 \mathrm{~min}$ respectively. The last motion field is the difference $\tilde{\mathbf{v}}^{k}(2 h 30)-\tilde{\mathbf{v}}^{k}(1 h 15)$ and illustrates the temporal changes. 


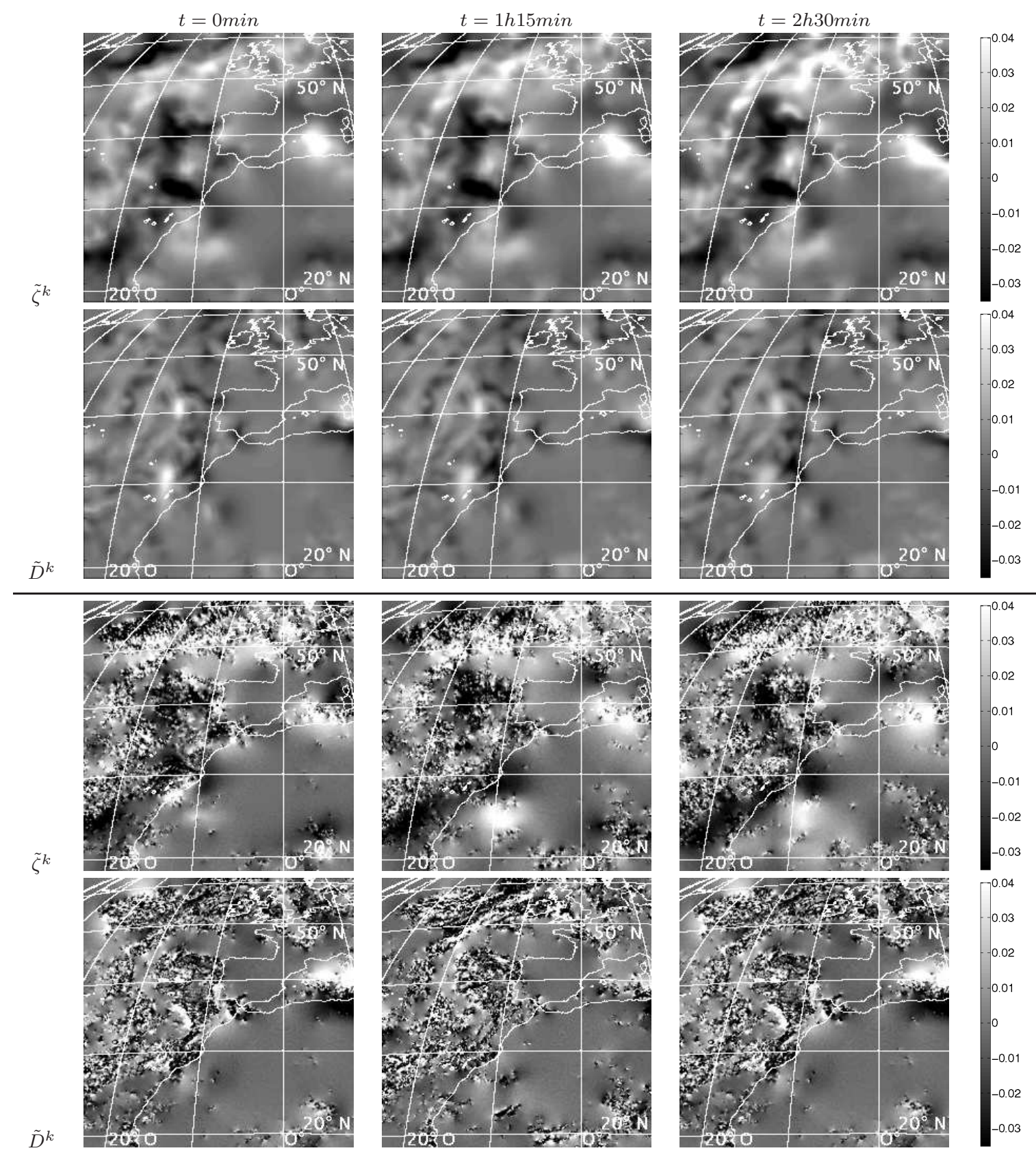

Fig. 5. Comparison of results from assimilation (top) and optical-flow (bottom) for the lower layer at $t=0 \mathrm{~min}, t=1 \mathrm{~h} 15 \mathrm{~min}$ and $t=2 h 30$ min respectively. The first line represents the vorticity and the second line the divergence. One can conclude that both estimations are in accordance but the one from the assimilation has a better temporal consistency. 